\title{
Artillery Shells over Circinus
}

\author{
Sylvain Veilleux ${ }^{1}$ and Jonathan Bland-Hawthorn ${ }^{2}$
}

Received —_; accepted

\footnotetext{
${ }^{1}$ Department of Astronomy, University of Maryland, College Park, MD 20742; E-mail: veilleux@astro.umd.edu

${ }^{2}$ Anglo-Australian Observatory, P.O. Box 296, Epping, NSW 2121, Australia; E-mail: jbh@aaossz.aao.gov.au
} 


\begin{abstract}
The recently identified Circinus Galaxy is the nearest ( $\sim 4 \mathrm{Mpc})$ Seyfert 2 galaxy known and we now demonstrate to be one of the best laboratories for studying the effects of nuclear activity|3 on the surrounding environment. Here we present new imaging Fabry-Perot observations of Circinus which confirm the existence of an ionization cone in this object but also show for the first time a complex of ionized filaments extending radially from the nucleus out to distances of $1 \mathrm{kpc}$. Arcs suggestive of bow shocks are observed at the terminus of some of these filamentary structures. Most spectacular of all, one of the structures appears to be a scaled-up version of a Herbig-Haro jet. The velocity field of the filaments confirms that they represent material expelled from the nucleus (possibly in the form of 'bullets') or entrained in a wide-angle wind roughly aligned with the polar axis of the galaxy. The motions observed across the ionization cone are highly supersonic, so high-velocity shocks are likely to contribute to the ionization of the line emitting gas. However, it is not clear at present whether shock ionization dominates over photoionization by the Seyfert 2 nucleus. Extrapolation of the filaments to smaller radii comes to within 1" (about 20 pc) of the infrared nucleus, therefore suggesting a AGN or nuclear starburst origin to these features. The complex of radial filaments detected in the Circinus galaxy is unique among active galaxies. The frequency of such events is unknown since only a handful of galaxies have been observed at the sensitivity level of our present observations. The event in the Circinus galaxy may represent a relatively common evolutionary phase in the lives of gas-rich active galaxies during which the dusty cocoon surrounding the nucleus is expelled by the action of jet or wind phenomena.
\end{abstract}

\footnotetext{
${ }^{3}$ In this paper, we use the terms 'nuclear activity' to refer to either starburst or black-hole driven activity in the nuclei of galaxies. Similarly, we refer to 'active galaxies' as galaxies powered by star formation (starburst galaxies) or through accretion onto a massive black hole (active galactic nuclei).
} 
Subject headings: galaxies: active — galaxies: individual (Circinus) — galaxies: jets — galaxies: kinematics and dynamics — galaxies: nuclei — galaxies: Seyfert — galaxies: starburst 


\section{Introduction}

The Circinus galaxy is a large, isolated, gas-rich spiral seen through a relatively unobscured $\left(A_{V} \approx 1.5 \mathrm{mag}\right)$ optical window near the Galactic plane $\left(b=-3.8^{\circ}\right.$; Lyngo \& Hansson 1972 ; Mebold et al. 1976; Freeman et al. 1977). The early detection of strong radio emission from the nucleus (Freeman et al. 1977) provided the first evidence for nuclear activity in this galaxy. Recent maps (Harnett et al. 1990; Elmouttie et al. 1995) have resolved spectacular radio lobes centered on the nucleus, and extending more than $90^{\prime \prime}(\sim 2 \mathrm{kpc})$ on either side of the galaxy disk $\left(\mathrm{PA} \approx-60^{\circ}\right)$. Additional evidence of unusual nuclear activity is suggested by the discovery of an intense 22- $\mathrm{GHz} \mathrm{H}_{2} \mathrm{O}$ megamaser (Gardner \& Whiteoak 1982; Whiteoak \& Gardner 1986). Detailed spectroscopic studies at optical and infrared wavelengths have since revealed the presence of a Seyfert 2 nucleus surrounded by an extended (200-pc radius) circumnuclear starburst (Oliva et al. 1994, 1995; Ghosh 1992; Marconi et al. 1995). A one-sided ionization cone and gas motions indicative of a large central mass concentration have recently been discovered in the Circinus galaxy (Marconi et al. 1995; Greenhill et al. 1997), making it the closest galaxy where the engine responsible for the nuclear activity can convincingly be attributed to a supermassive black hole surrounded by a thick obscuring screen. In this Letter, we describe new TAURUS-2 and long-slit spectroscopic observations which reveal a complex of radial filaments emanating from the nucleus of this galaxy. We propose two possible scenarios to explain these features and briefly discuss the implications of our new results.

\section{Observations}

The Circinus galaxy was observed for 165 minutes on the night of February 211995 using the TAURUS-2 imaging Fabry-Perot interferometer at the AAT 3.9-m telescope. This instrument was used in the Angstrom imaging mode to maximize sensitivity to faint line emission (Bland-Hawthorn et al. 1997). A $40 \mu \mathrm{m}$ gap etalon was used out of band to produce deep, low-resolution ( $~ 350$ $\mathrm{km} \mathrm{s}^{-1}$ sampled at every $\sim 30 \mathrm{~km} \mathrm{~s}^{-1}$ ) [O III] $\lambda 5007$ spectra at each point across this galaxy. 
Each square pixel subtended $0{ }^{\prime \prime} 315$ on the sky; the atmospheric seeing at FWHM averaged approximately 4 times this value. These data comprise kinematic and photometric information for the $\left[\mathrm{O}\right.$ III] line at $\sim 10,000$ positions over the central $2^{\prime}$ of the Circinus galaxy. At a later stage, Circinus was observed at several other etalon spacings to derive the distribution of $\mathrm{H} \alpha$ emission and construct line profiles of [S II] $\lambda \lambda 6716,6731$ and [O III $] \lambda 5007$ at $1 \AA$ resolution. Narrow-band filters were also used at different tilt angles to isolate the lines of He II $\lambda 4686$, [S II] $\lambda \lambda 6717,6731$ and $\mathrm{H} \alpha$, and in order to subtract neighbouring continuum emission. The $\mathrm{H} \alpha$ flux map is discussed below, but the rest of these data are to be presented in a more detailed study (in progress).

In addition, S.L. Lumsden kindly obtained long-slit observations for us at two positions in Circinus. The RGO spectrograph on the AAT was used at the Cassegrain $\mathrm{f} / 8$ focus with the $25 \mathrm{~cm}$ camera and the $270 \mathrm{R}$ grating. At a plate scale $0^{\prime \prime} 77 \mathrm{pix}^{-1}$, this set up gave a resolution of $3.4 \AA$ FWHM at $\mathrm{H} \alpha$ and a wavelength coverage of $4770-8260 \AA$. The $2^{\prime \prime}$ slit was somewhat oversized compared to the 1". 1 FWHM seeing. Both slits were aligned at a position angle of $270^{\circ}$, one passing through the nucleus, the other offset $6 . \prime 0 \mathrm{~N}$ of the nucleus. The exposure time for both positions and an offset sky position was 600 sec.

\section{Results}

Figures 1 and 2 show the [O III] and $\mathrm{H} \alpha$ line flux images obtained by integrating the line profiles in the Fabry-Pero data. Only the blueshifted (between -150 and $0 \mathrm{~km} \mathrm{~s}^{-1}$ ) H $\alpha$ emission is presented in these figures to minimize contamination from the (redshifted) circumnuclear emission south-west of the nucleus. These data confirm the existence of a bright ionization cone in Circinus (Marconi et al. 1995), an effect also apparent in our He II and [S II] narrow-band images. But the [O III] data also reveal a number of fainter filaments beyond this cone which cannot be explained by simple illumination effects of an homogeneous environment by an anisotropic (biconical) source of radiation in the nucleus.

The most striking [O III] feature extends along position angle $\sim-50^{\circ}$ spanning a distance of 
$\sim 25-45^{\prime \prime}(500-900 \mathrm{pc})$ from the nucleus. The lateral extent of this filament is near the limit of our resolution ( $\sim 1^{\prime \prime} .5$ after smoothing). This narrow feature is also visible at $\mathrm{H} \alpha$ but with a lower contrast. The gas at this location is highly ionized with a [O III] $\lambda 5007 / \mathrm{H} \alpha$ flux ratio typically larger than unity. Extrapolation of this filament to smaller radii comes to within $1^{\prime \prime}$ (20 pc) of the infrared nucleus (Marconi et al. 1995), suggesting a nuclear (AGN or compact starburst) origin to this feature. A second [O III] filament is also detected, emerging from the nucleus along PA $\approx$ $-120^{\circ}$ out to a maximum radius of $\sim 35^{\prime \prime}(700 \mathrm{pc})$. These radial filaments resemble the optical counterparts of radio jets in more powerful active galaxies (e.g., Sparks, W. B., Biretta, J. A., \& Macchetto, F. 1994).

The most spectacular feature in the $\mathrm{H} \alpha$ data is the hook-shaped filament which extends to $40^{\prime \prime}(800 \mathrm{pc})$ west of the nucleus (Fig. 2). Such features are commonly observed in Herbig-Haro (HH) objects (e.g., HH 47; Hartigan et al. 1993) although have never been seen on galactic scales. The western 'hook' is far more elongated than the wind-blown bubbles in M82 (Bland \& Tully 1988) and NGC 3079 (Veilleux et al. 1994). Additional morphological evidence for outflow exists in the northern portion of our data (Fig. 1). The [O III ] emission along PA $\approx-20^{\circ}$ forms a broad filamentary 'finger' or jet that points back to the nucleus. A knot is present at the tip of this 'finger', $25^{\prime \prime}$ from the nucleus. Bright $\mathrm{H} \alpha$ emission is also visible near this position, the southern portion of which forms a wide $\left(\sim 8^{\prime \prime}\right)$ arc resembling a bow shock. The arc is pointing in the downstream direction consistent with being produced by a collimated jet.

The kinematics derived from the [O III] Fabry-Perot data (Fig. 3) and long-slit spectra (Figs. $4 \& 5)$ bring credence to this nuclear outflow scenario. Non-gravitational motions are observed throughout the [O III] cone, superposed on a large-scale velocity gradient caused by galactic rotation along the major axis of the galaxy $\left(\mathrm{PA}_{\text {maj }} \approx 30^{\circ}\right.$; Freeman et al. 1977). An unusually large velocity gradient of $4 \mathrm{~km} \mathrm{~s}^{-1} \mathrm{pc}^{-1}$ is seen near the position of the bright knot $\sim 12^{\prime \prime}$ from the nucleus along $\mathrm{PA} \approx-30^{\circ}$ (Fig. 3). The side of the knot facing the nucleus presents velocities that are nearly $250 \mathrm{~km} \mathrm{~s}^{-1}$ lower than gas only $3^{\prime \prime}$ north of that position. The emission profiles near that knot are broad $\left(\sim 250 \mathrm{~km} \mathrm{~s}^{-1}\right)$ and perhaps complex. 
The material in the brighter portions of the NW and SW filaments does not seem to take part in the galactic rotation (Fig. 3). The velocities in the NW [O III] filament appear systematically blueshifted by $\sim 0-100 \mathrm{~km} \mathrm{~s}^{-1}$ with respect to the systemic velocity (439 $\mathrm{km} \mathrm{s}^{-1}$; Freeman et al. 1977), while the velocities of the gas in the SW filament are roughly systemic within the errors of the measurements. Non-gravitational motions are also detected along the western 'hook' feature (Fig. 4). A velocity gradient of $80 \mathrm{~km} \mathrm{~s}^{-1}$ over $8^{\prime \prime}\left(0.5 \mathrm{~km} \mathrm{~s}^{-1} \mathrm{pc}^{-1}\right)$ is visible near the location of knot 2 (Fig. 4a). Perhaps the strongest evidence for shocks in our data is seen in knot 4. There, the strong $\mathrm{H} \alpha$ line is blueshifted by $180 \mathrm{~km} \mathrm{~s}^{-1}$ with respect to the [NII] doublet (Figs. 4b \& 5). Velocity shifts between different emission lines are frequently observed in $\mathrm{HH}$ objects and reflect spatially distinct line-emitting regions (e.g., leading edge versus cooling tail; Morse et al. 1994 and references therein). A more detailed analysis of the nuclear long-slit spectra also suggests the presence of broad $\left(\mathrm{FWZI} \approx 800 \mathrm{~km} \mathrm{~s}^{-1}\right.$ ) blueshifted wings in the emission-line profiles produced by knots 2 and 3, but this result needs to be confirmed with spectra of higher signal-to-noise ratio and velocity resolution.

The current radio data also support the existence of a wide-angle outflow in the Circinus galaxy. The NW feature appears to have a radio counterpart at both $13 \mathrm{~cm}$ and $20 \mathrm{~cm}$ (the NW 'plume' in Figs. 2-4 of Elmouttie et al 1995). This appears as a continuum ridge which runs $\mathrm{SE}-\mathrm{NW}$ through the radio map out to $90^{\prime \prime}$ in radius within the bisymmetric lobes. Radio jets in active galaxies are commonly observed to have associated optical emission, but normally confined to an outer surface at the terminus of the shock (Cecil, Bland, \& Tully 1990). The radio data do not have sufficient resolution to argue whether the optical emission fills the NW 'plume' or is confined to the shock front. The $13 \mathrm{~cm}$ radio map also shows a narrow feature which extends due west from the nucleus. At higher resolution, we anticipate that this feature is associated with another jet - distinct from the NW jet - responsible for the western optical 'hook'. Any possible radio counterparts to the SW filament and the northern 'finger' are being masked by strong emission from the galactic disk.

The motions observed across the ionization cone are highly supersonic, so high-velocity 
$\left(V_{s} \gtrsim 100 \mathrm{~km} \mathrm{~s}^{-1}\right)$ shocks are likely to contribute to the ionization of the line emitting gas. Table 1 summarizes the line ratios measured from our long-slit spectra in knots $1-4$ of the western 'hook' and in the nucleus. Large variations are sometimes observed within a single knot. In knot 2, for instance, $[\mathrm{O} \mathrm{I}] / \mathrm{H} \alpha$ falls with radius as $[\mathrm{O} \mathrm{III}] / \mathrm{H} \beta$ increases. This gradient argues against photoionization (of ionization-bounded clouds) by the nucleus, unless the ionization parameter is rising due to a rapidly falling density profile. The relatively constant density-sensitive [S II] $\lambda 6717 / \lambda 6731$ ratio measured at these locations (especially at the two eastern positions in knot 2 ; cf. Table 1, off-nuclear spectrum) rules out this possibility. The very different excitation properties of knots 3 and 4 (cf. Table 1, nuclear spectrum; Fig. 5) are also difficult to explain in the pure nuclear photoionization scenario, although here large variations of the ionization parameter associated with density variations cannot formally be excluded because the $[\mathrm{S} \mathrm{II}] \lambda 6716 / \lambda 6731$ ratio is in the low-density limit and therefore cannot be used as a density indicator. The enhanced $[\mathrm{N} \mathrm{II}] / \mathrm{H} \alpha,[\mathrm{S} \mathrm{II}] / \mathrm{H} \alpha$, and $[\mathrm{O} \mathrm{III}] / \mathrm{H} \beta$ ratios in knots 1 and 2 fall near the range produced by the high velocity photoionizing radiative shocks of Dopita \& Sutherland (1995). However, [O I]/H $\alpha$ is considerably weaker than predicted by the models. Moreover, all of these ratios require large gas velocities $\left(\mathrm{V}_{s} \gtrsim 500 \mathrm{~km} \mathrm{~s}^{-1}\right)$ and therefore imply large projection effects to explain the relatively small apparent velocities in our data. Hybrid models involving fast shocks and power-law photoionization by the Seyfert 2 nucleus (e.g., Viegas-Aldrovandi \& Contini 1989 and references therein) alleviate these problems. Photoionization by the active nucleus of a mixture of ionization and matter-bounded clouds whose relative proportions vary with position in the galaxy may also provide another explanation for the abrupt changes of excitation in the filaments (e.g., Binette, Wilson, \& Storchi-Bergmann 1996).

\section{Origin of the Complex of Radial Filaments}

Jets in active galactic nuclei are attributed to gas centrifugally accelerated along magnetic field lines tied at one end to the accretion disks (Lynden-Bell 1996). The existence of several jets in a single galaxy is difficult to explain in these models unless the active galaxy is host to two or more 
black holes each possessing its own accretion disk and radio jet. A more plausible explanation for the optical filaments and the linear structures observed in the radio map is that they arise from individual mass structures ejected in a wide opening angle possibly from an explosive nuclear event. The apparent extent and velocities of the outflowing gas in the Circinus galaxy suggest that the purported explosive event took place a few million years ago. It is not clear what triggered this event since the Circinus galaxy shows no sign of recent galactic interaction (Freeman et al. 1977). The ejecta from this event have been funneled into a fan with opening angle of $\sim 100^{\circ}$ and symmetry axis along $\mathrm{PA} \approx-65^{\circ}$, i.e. along the direction of the NW radio lobe (Elmouttie et al. 1995). The lack of any optical counterpart to the SE radio lobe argues that the outflow on that side is hidden from view by the inclined $\left(\sim 65^{\circ}\right.$; Freeman et al. 1977$)$ galaxy disk. The symmetry axis of the conical outflow is roughly aligned with the minor axis of the galaxy, and therefore suggests that our line of sight lies only $\sim 15^{\circ}$ outside the cone defined by the outflow.

Hydrodynamical instabilities in the dense shell swept up by an expanding plasmon (Pedlar, Dyson, \& Unger 1985; Taylor et al. 1989) or a time-variable wind (Garcia-Segura, MacLow, \& Langer 1996; Stone, Xu, \& Mundy 1995) offer an alternative explanation for the optical morphology of the Circinus galaxy. Dynamical models (MacLow, McCray, \& Norman 1989; Tomisaka \& Ikeuchi 1988; Suchkov et al. 1994) of wind-blown bubbles have had success reproducing the range of phenomena observed in radio-lobe spirals (Wehrle \& Morris 1988; Wrobel 1994; Veilleux et al. 1994). The outflow event in the Circinus galaxy would fundamentally be driven by this same mechanism, but would correspond to a later stage of evolution when the initial wind-blown shell has broken out and left dense clouds that have since been accelerated and stretched radially by the galactic wind (free wind phase). The impact of a high-speed supersonic wind on dense clouds has been discussed in several contexts including shock-induced star formation (Woodward 1976), supernova blast waves (McKee et al. 1987), and broad-absorption line quasars (Schiano, Christiansen, \& Knerr 1995). Numerical simulations indicate that the initial encounter of the clouds with the wind medium drives a strong shock that may have devastating effects on the cloud structure (Woodward 1976; McKee et al. 1987). Once in ram pressure equilibrium with the wind, however, these clouds may be accelerated up to a significant fraction of the wind velocity before 
Rayleigh-Taylor and Kelvin-Helmholtz instabilities tear them apart (Schiano et al. 1995).

A clue to the origin of the outflow in the Circinus galaxy is provided by the energetics of this event. The mass taking part in this outflow can be estimated from the [O III] $\lambda 5007$ flux. We parametrize the mass in terms of the density, which is poorly constrained, and use an electron

temperature of $10^{4} \mathrm{~K}$. The integrated observed [O III] intensity of $\sim 5 \times 10^{-13} \mathrm{erg} \mathrm{s}^{-1} \mathrm{~cm}^{-2}$ implies a mass of a few times $10^{4} \mathrm{X}^{-1} \mathrm{~N}_{e, 2}^{-1} \mathrm{M}_{\odot}$ where $\mathrm{X}$ is the fraction $(<1)$ of oxygen which is doubly ionized and $\mathrm{N}_{e, 2}$ is the electron density in units of $10^{2} \mathrm{~cm}^{-3}$. In this calculation, we used $\mathrm{A}_{v}=1.5 \mathrm{mag}$, a solar mass fraction for oxygen, and a five-level atom approximation to estimate the emission coefficient (McCall 1984). Taking $200 \mathrm{~km} \mathrm{~s}^{-1}$ as representative for the deprojected gas velocities (a uncertain number; cf. last paragraph of $\S 3$ ), the kinetic energy involved in the NW outflow is a few times $10^{52} \mathrm{~N}_{e, 2}^{-1}$ ergs. A value closer to $\sim 10^{53} \mathrm{~N}_{e, 2}^{-1}$ ergs is probably more representative of the total energy involved in the outflow event since the radio data (Harnett et al. 1990; Elmouttie et al. 1995) suggest that a similar mass outflow is also taking place on the SE side of the nucleus. The energetics of the optical outflow in the Circinus galaxy therefore appear to be relatively modest (equivalent to about 100 supernova explosions if $\mathrm{N}_{e} \approx 100 \mathrm{~cm}^{-3}$ ) and appear to lie at the low energy end of the distribution for wide-angle events observed in nearby galaxies (Cecil et al. 1990; Bland \& Tully 1988; Heckman et al. 1990; Veilleux et al. 1994). This outflow can easily be powered by the AGN or by a compact nuclear starburst. The nuclear starburst detected in Circinus (Oliva et al. 1995) appears somewhat older than the present outflow event, however.

\section{Summary and Implications}

Deep imaging Fabry-Perot data reveal a complex of radial line-emitting filaments in the Circinus galaxy, the closest Seyfert 2 galaxy known. The kinematics of the gas producing these features suggest the ejection of material over a wide opening angle or inhomogeneities in a wide-angle outflow. The proximity of the Circinus galaxy makes it a unique laboratory to study with unprecedented resolution the impact of nuclear winds, supersonic ejecta, and jets on the 
interstellar medium of galaxies. The apparent rarity of radial filaments and bow shocks in active galaxies may reflect the ephemeral nature of this phenomenon or the difficulty in accelerating dense gas clouds coherently. However, few galaxies have been observed with the sensitivity of our present observations. The discovery of these features in the Circinus galaxy, a spiral galaxy with an abnormal richness of gas (Freeman et al. 1977), brings up the possibility that we may be witnessing a common evolutionary phase in the lives of gas-rich active galaxies. Observations of other active galaxies at similar sensitivity will help establish the frequency and duration of this phenomenon. 
We acknowledge useful conversations with Roger Blandford, Pat Hartigan, and Jim Stone on the physics of outflows. We would particularly like to thank S. L. Lumsden for obtaining the long-slit spectra presented in this paper, Keith Jones for helpful comments on the radio data, Brent Tully for the loan of the HIFI etalon, and the referee, E. Oliva, for several suggestions which improved this paper. Parts of this work have been supported by NASA through grant number HF-1039.01-92A awarded by the Space Telescope Science Institute which is operated by the AURA, Inc. for NASA under contract No. NAS5-26555 (SV). 


\section{REFERENCES}

Binette, L., Wilson, A. S., \& Storchi-Bergmann, T. 1996, A\&A, 312, 365

Bland-Hawthorn, J., Meara, S.J.P., Taylor, K., \& Cannon, R.D. 1997, AJ, in press

Bland, J., \& Tully, R. B. 1988, Nature, 334, 43

Cecil, G., Bland, J., \& Tully, R. B. 1990, ApJ, 355, 70

Dopita, M.A., \& Sutherland, R.S. 1995, ApJ, 455, 468

Elmouttie, M., et al. 1995, MNRAS, 275, L53

Freeman, K. C., et al. 1977, A\&A, 55, 445

García-Segura, G., MacLow, M.-M., \& Langer, N. 1996, A\&A, 305, 229

Gardner, F. F., \& Whiteoak, J. B. 1982, MNRAS, 201, 13p

Ghosh, S. K. 1992, ApJ, 391, 111

Greenhill, L. J., et al. 1997, ApJ, 474, L103

Harnett, J. I., et al. 1990, MNRAS, 244, 130

Hartigan, P., Morse, J.A., Heathcote, S., \& Cecil, G. 1993, ApJ, 414, L121

Heckman, T. M., Armus, L., \& Miley, G. K. 1990, ApJS, 74, 833

Lynden-Bell, D. 1996, MNRAS, 279, 389

Lyngo, G., \& Hansson, N. 1972, A\&A Suppl., 6, 327

MacLow, M.-M., McCray, R., \& Norman, M. 1989, ApJ, 337, 141

Marconi, A., Moorwood, A. F. M., Origlia, L., \& Oliva, E. 1995, ESO Mess., 78, 20

McCall, M. L. 1984, MNRAS, 208, 253

McKee, C. F., Hollenbach, D. J., Seab, C. G., \& Tielens, A. G. G. M. 1987, ApJ, 318, 674

Mebold, U., Goss, W. M., van Woerden, H., \& Freeman, K. C. 1976, Proc. astr. Soc. Aust., 3, 72

Morse, J. A., Hartigan, P., Heathcote, S., Raymond, J. C., \& Cecil, G. 1994, ApJ, 425, 738 
Oliva, E., Origlia, L., Kotilainen, J. K., \& Moorwood, A. F. M. 1995, A\&A, 301, 55

Oliva, E., Salvati, M., Moorwood, A.F.M, \& Marconi, A. 1994, A\&A, 288, 457

Pedlar, A., Dyson, J.E., \& Unger, S.W. 1985, MNRAS, 214, 463

Schiano, A. V. R., Christiansen, W. A., \& Knerr, J. M. 1995, ApJ, 439, 237

Sparks, W. B., Biretta, J. A., \& Macchetto, F. 1994, ApJS, 90, 909

Stone, J. M, Xu, J., \& Mundy, L G. 1995, Nature, 377, 315

Suchkov, A. A., Balsara, D. S., Heckman, T. M., \& Leitherer, C. 1994, ApJ, 430, 511

Taylor, D., Dyson, J.E., Axon, D.J., \& Pedlar, A. 1989, MNRAS, 240, 487

Tomisaka, K., \& Ikeuchi, S. 1988, ApJ, 330, 695

Veilleux, S., et al. 1994, ApJ, 433, 48

Viegas-Aldrovandi, S. M., \& Contini, M. 1989, ApJ, 339, 689

Wehrle, A. E., \& Morris, M. 1988, AJ, 95, 1689

Whiteoak, J. B., \& Gardner, F. F. 1986, MNRAS, 222, 513

Woodward, P. R. 1976, ApJ, 207, 484

Wrobel, J. M. 1994, BAAS, 26, 1501 
Fig. 1.- Line flux images of the Circinus galaxy: $a$, [O III] $\lambda 5007$ and $b$, blueshifted (between -150 and $\left.0 \mathrm{~km} \mathrm{~s}^{-1}\right) \mathrm{H} \alpha$. North is at the top and west to the right. The position of the infrared nucleus (Marconi et al. 1995) is indicated in each image by a cross. The spatial scale, indicated by a horizontal bar at the bottom of the [O III] image, is the same for each image and corresponds to $\sim 25^{\prime \prime}$ or $500 \mathrm{pc}$ for the adopted distance of the Circinus galaxy of $4 \mathrm{Mpc}$. The minor axis of the galaxy runs along $\mathrm{PA} \approx 60^{\circ}$ (measured from north to east). The faintest features in the [O III] $(\mathrm{H} \alpha)$ image have a surface brightness of $\sim 4(20) \times 10^{-17} \mathrm{erg} \mathrm{s}^{-1} \mathrm{~cm}^{-2} \operatorname{arcsec}^{-2}$. To suppress the wide dynamic range, the intensity greyscale wraps around and becomes logarithmic at high intensity levels. The radial features along the north (labelled N), north-west, west (labelled W), and south-west axes suggest the ejection of material (possibly in the form of 'bullets') over a wide opening angle or inhomogeneities in a wide-angle outflow. The inclined $\left(\sim 65^{\circ}\right.$; Freeman et al. 1977) galaxy disk hides the south-east portion of this outflow.

Fig. 2.- Line flux images of the western hook-shaped filament: $a$, [O III] $\lambda 5007$ and $b$, blueshifted (between -150 and $0 \mathrm{~km} \mathrm{~s}^{-1}$ ) $\mathrm{H} \alpha$. The position of the infrared nucleus (Marconi et al. 1995) is indicated in each image by a cross. The orientation is the same as in Fig. 1, but the horizontal bar at the bottom of the [O III] image now corresponds to $\sim 250 \mathrm{pc}$. The faintest features in the [O III] $(\mathrm{H} \alpha)$ image have a surface brightness of $\sim 1(4) \times 10^{-16} \mathrm{erg} \mathrm{s}^{-1} \mathrm{~cm}^{-2} \operatorname{arcsec}^{-2}$. Once again, the intensity greyscale wraps around and becomes logarithmic at high intensity levels. The hookshaped filament shares a strong resemblance with HH objects produced by young stars, suggesting a bow-shock origin for this feature. The bow-shock terminus (labelled 4 in the figure) is only detected in $\mathrm{H} \alpha$, but a rough correspondence is observed between the [O III] and $\mathrm{H} \alpha$ knots (labelled $1-3$ ) which delineate the northern edge of this structure. 
Fig. 3.- Velocity field derived from the [OIII] data cube. The velocities range from $170 \mathrm{~km}$ $\mathrm{s}^{-1}$ (white) to $650 \mathrm{~km} \mathrm{~s}^{-1}$ (black). The uncertainties range from about $50 \mathrm{~km} \mathrm{~s}^{-1}$ in the bright line-emitting regions to $100 \mathrm{~km} \mathrm{~s}^{-1}$ or more in the fainter areas. The position of the infrared nucleus (Marconi et al. 1995) is indicated by a cross. The orientation is the same as in Fig. 1, but the horizontal bar at the bottom of the image now corresponds to $\sim 250 \mathrm{pc}$. The large-scale velocity gradient along $\mathrm{PA} \approx 30^{\circ}$ is due to galactic rotation. Superposed on this gradient are non-gravitational motions observed at several locations, including the NW, W, and SW filaments visible in Figure 1 , and a compact region $\sim 12^{\prime \prime}$ from the nucleus along $\mathrm{PA} \approx-30^{\circ}$ coinciding with very strong [O III] emission.

Fig. 4.- Sky-subtracted long-slit spectra. (a) 6!'0 north of nucleus, PA $=270^{\circ}$, (b) through nucleus, $\mathrm{PA}=270^{\circ}$. The vertical bar on the right of each panel represents $\sim 250$ pc. The offnuclear spectrum intersects knots 1 and 2 of Fig. 2, while the nuclear spectrum intersects knots 3 and 4 but only the southern portions of knots 1 and 2. Note the velocity gradient in knot 2 of the upper panel and the strong blueshifted $\mathrm{H} \alpha$ emission in knot 4.

Fig. 5.- Binned spectra at four positions along the slit through the nucleus corresponding to knots 1-4. Each spectrum has been offset by 400 counts. Knots 1 and 2 are binned along the slit over $6^{\prime \prime}$; knots 3 and 4 are binned over $4^{\prime \prime}$. Note the greatly enhanced [N II]/H $\alpha$ ratio along the jet, except on the bow shock at knot 4. Furthermore, the $\mathrm{H} \alpha$ line at knot 4 is blueshifted by $180 \mathrm{~km}$ $\mathrm{s}^{-1}$ with respect to the $[\mathrm{N}$ II] lines. A faint blue wing can be seen on many of the emission lines. 


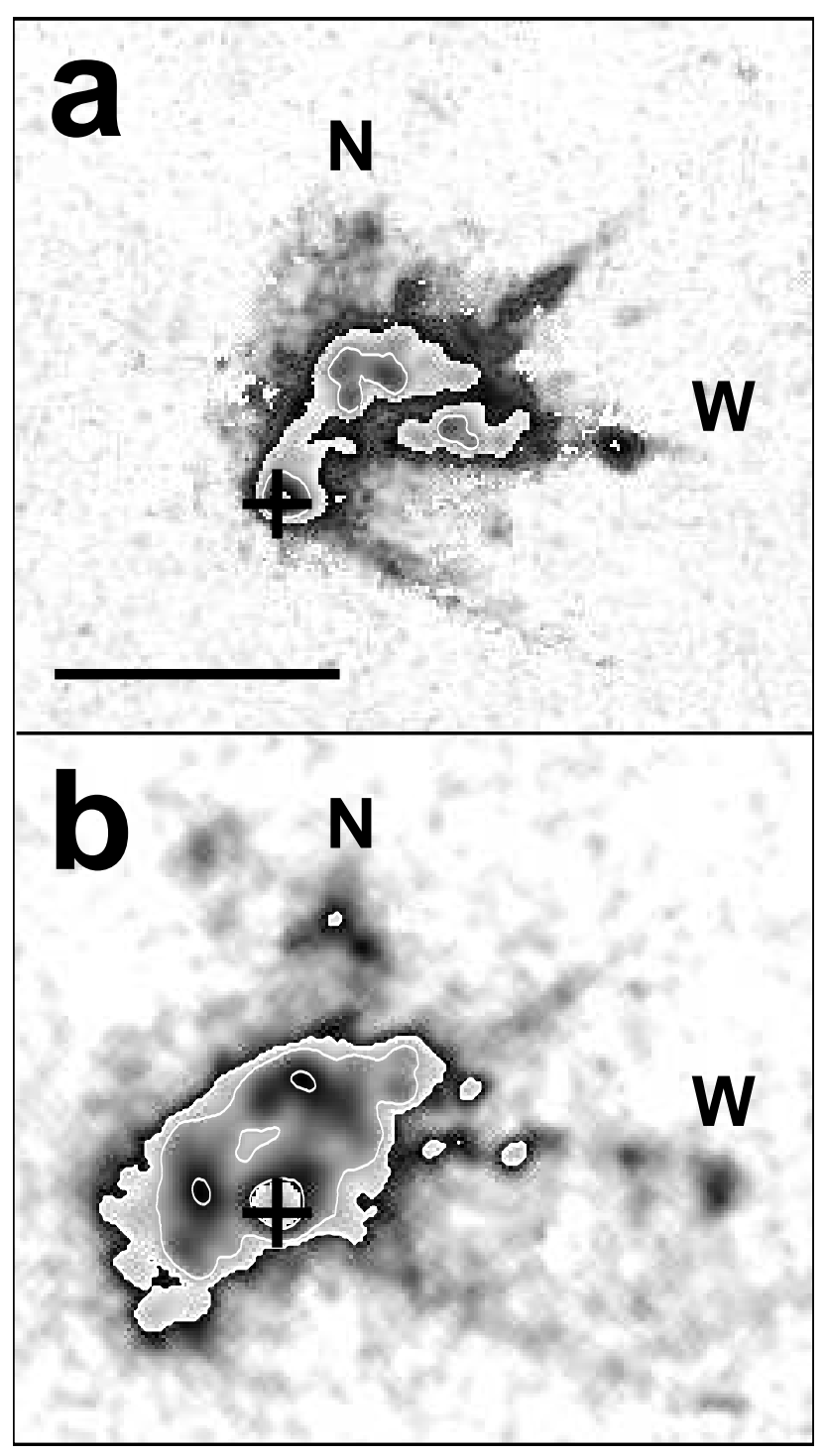

Fig. 1.- 


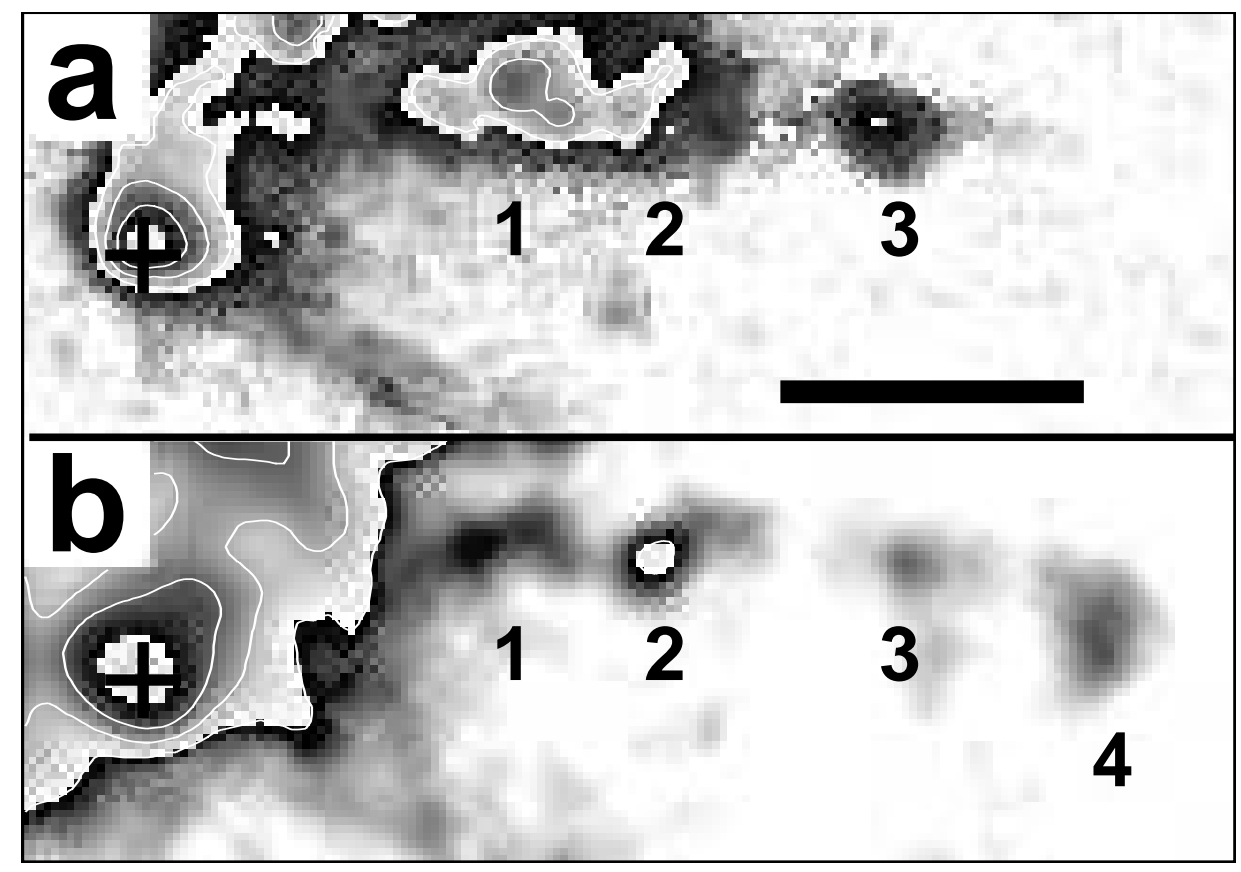

Fig. 2.- 


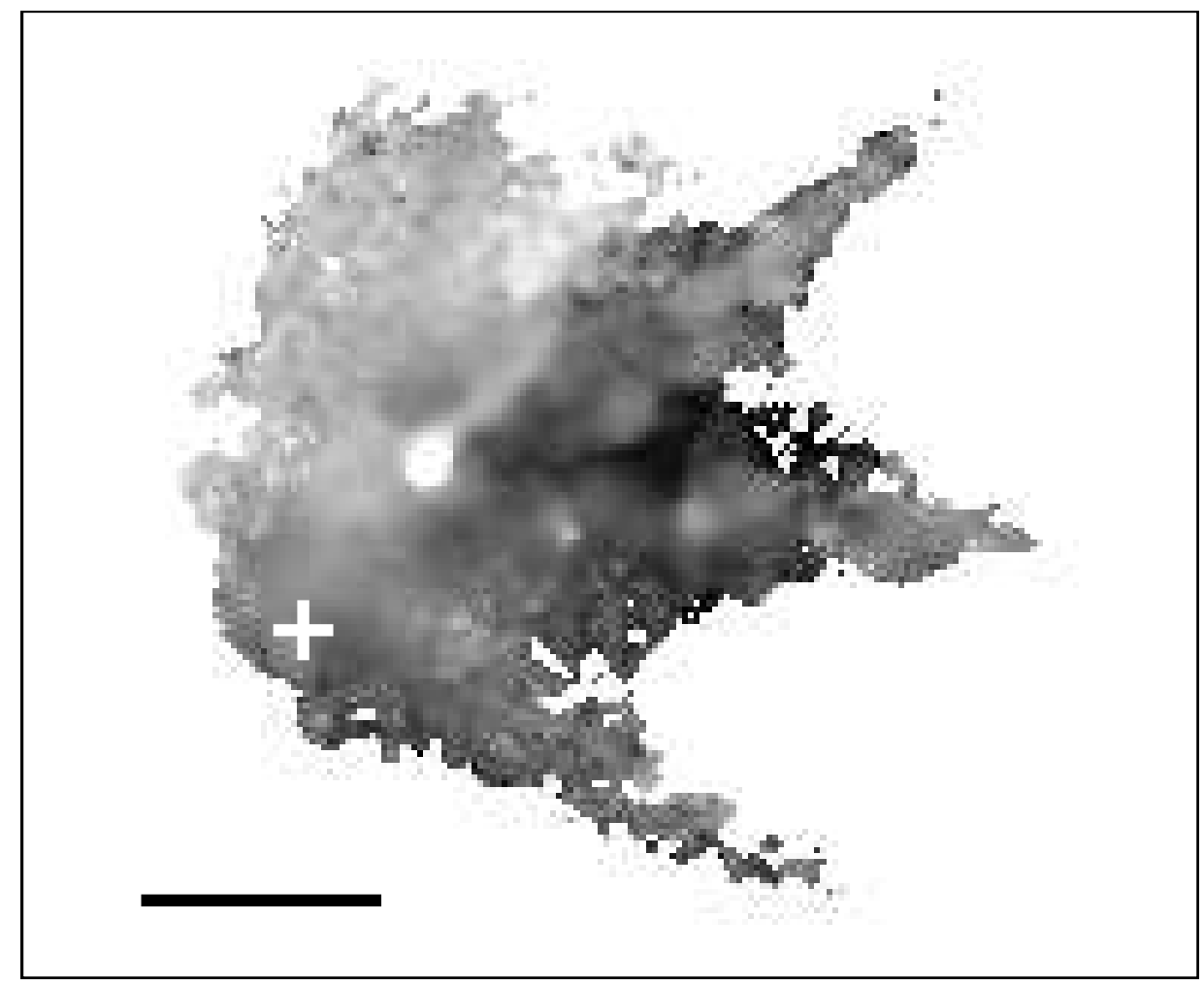

Fig. 3.- 


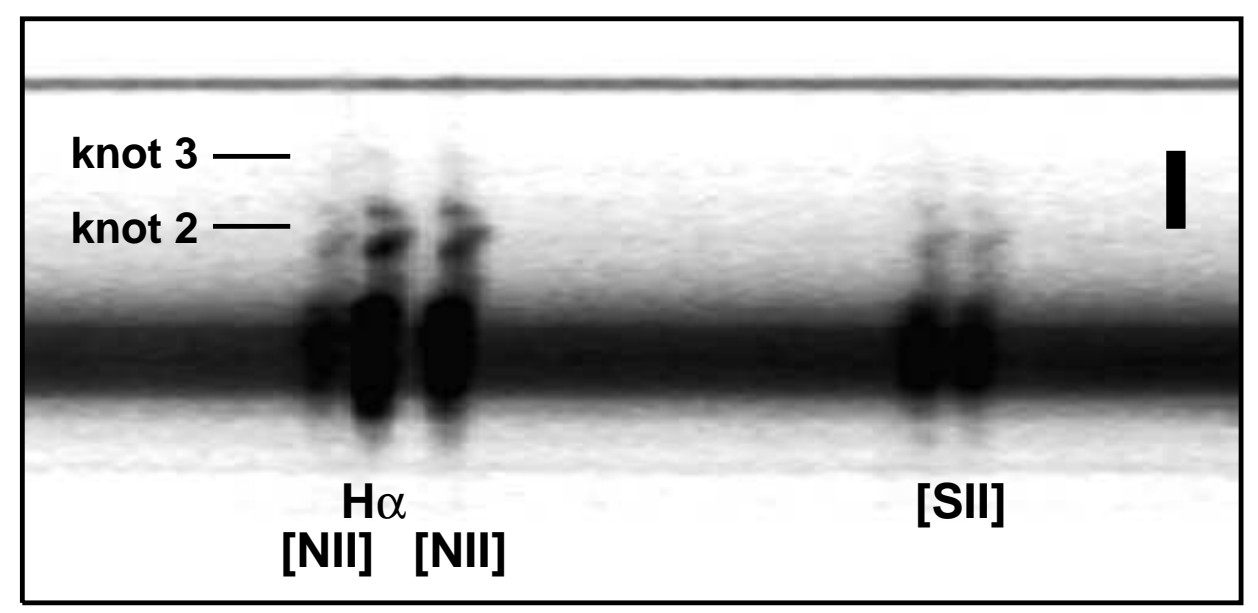

Fig. 4.- a.

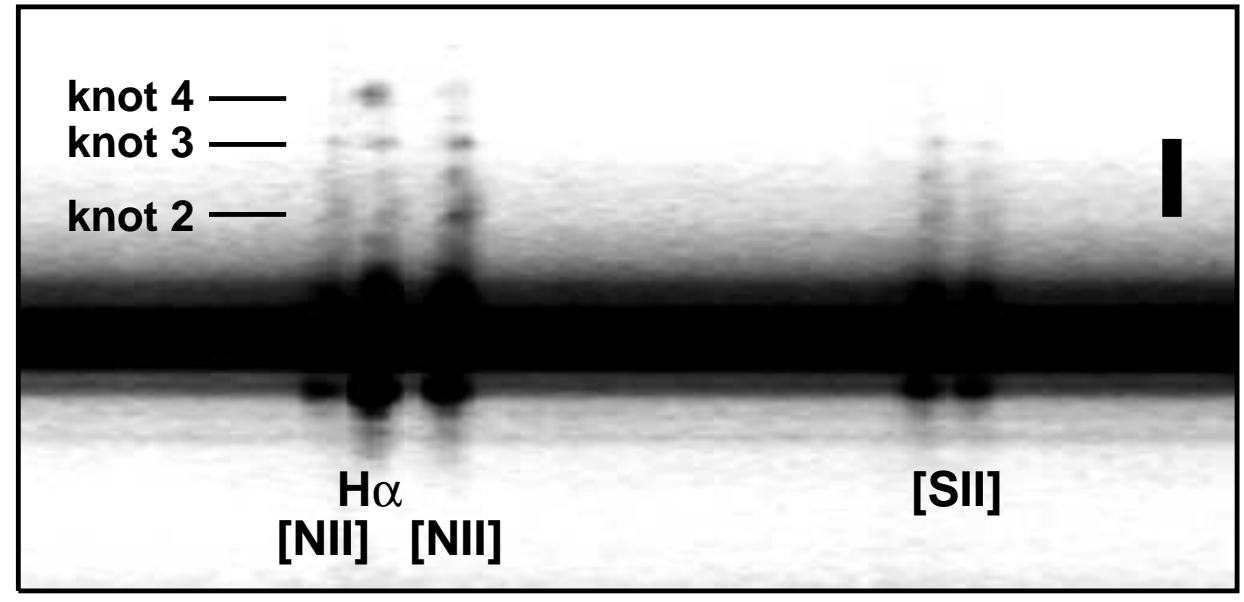

Fig. 4.- b. 


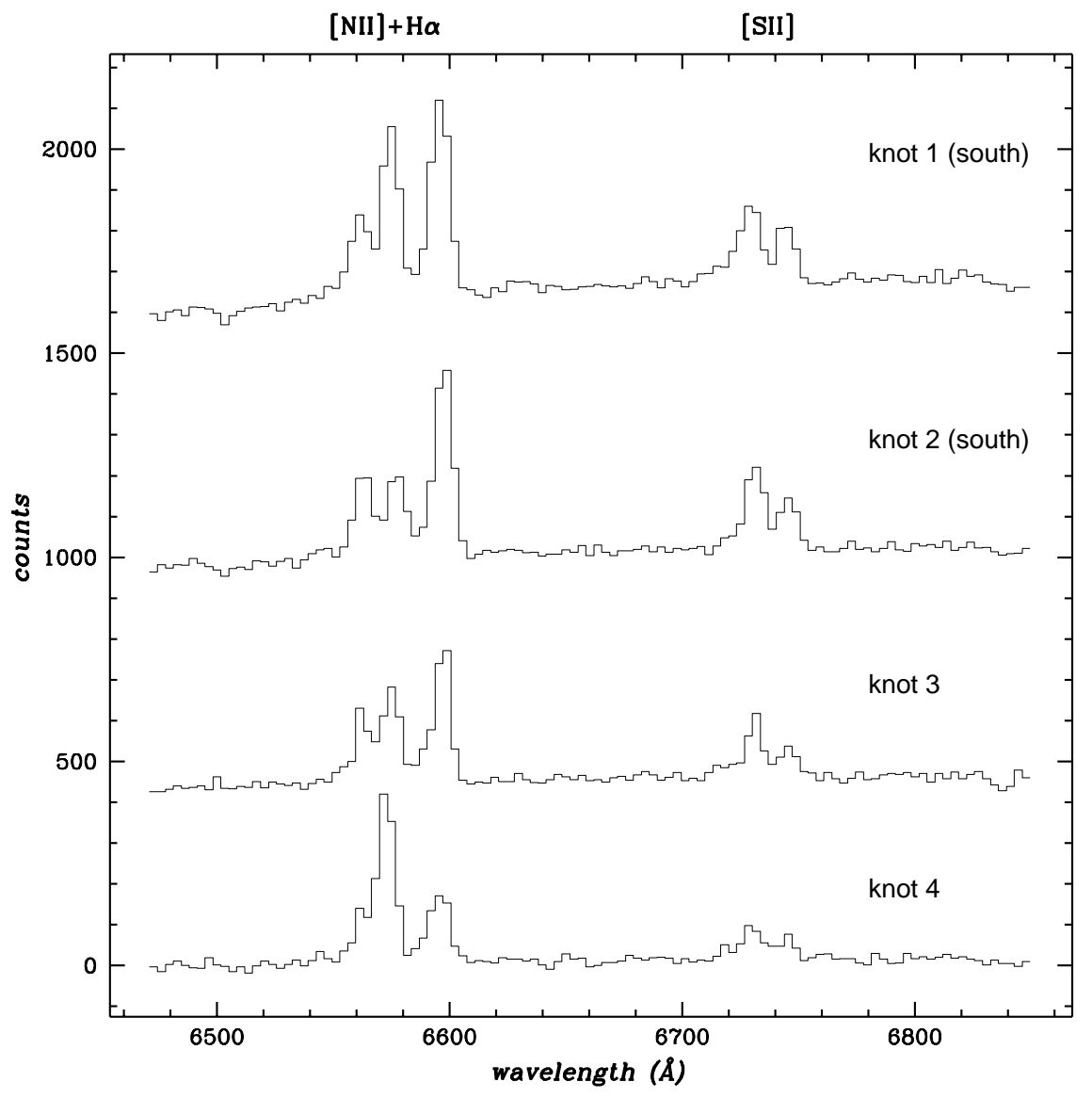

Fig. 5.- 
Table 1: Emission-Line Ratios derived from Long-Slit $\operatorname{Spectra}^{(1)}$.

\begin{tabular}{|c|c|c|c|c|c|c|}
\hline Feature & Region & {$[\mathrm{O} \quad \mathrm{III}] / \mathrm{H} \beta$} & {$[\mathrm{O} \mathrm{I}] / \mathrm{H} \alpha$} & {$[\mathrm{N}$ II $] / \mathrm{H} \alpha$} & {$[\mathrm{S} \mathrm{II}] / \mathrm{H} \alpha$} & [S II] $6717 / 6731$ \\
\hline$(1)$ & $(2)$ & (3) & $(4)$ & $(5)$ & (6) & (7) \\
\hline \multicolumn{7}{|c|}{ Off-Nuclear Spectrum: 6!'0 north of nucleus, PA $=270^{\circ}$} \\
\hline Disk & $-10.0-10.0$ & 8.94 & 0.23 & 0.84 & 0.60 & 1.38 \\
\hline Knot 1 & $10.8-13.9$ & 10.0 & 0.32 & 0.99 & 0.73 & 1.78 \\
\hline Knot 2 & $14.6-16.2$ & 13.9 & 0.23 & 0.89 & 0.58 & 1.22 \\
\hline Knot 2 & $16.9-20.0$ & 16.9 & 0.13 & 0.75 & 0.45 & 1.27 \\
\hline Knot 2 & $20.8-22.3$ & 17.2 & 0.13 & 0.97 & 0.59 & 1.54 \\
\hline \multicolumn{7}{|c|}{ Nuclear Spectrum: through nucleus, $\mathrm{PA}=270^{\circ}$} \\
\hline Nucleus & $-3.9-3.9$ & 13.9 & 0.12 & 1.12 & 0.28 & 1.20 \\
\hline Knot 1 & $11.6-17.7$ & $\ldots^{(2)}$ & 0.13 & 1.41 & 0.93: & 2.0 \\
\hline Knot 2 & $18.5-23.9$ & $\ldots{ }^{(2)}$ & $0.8:$ & 2.00 & 1.42 & 1.85 \\
\hline Knot 3 & $30.8-33.9$ & $\ldots{ }^{(2)}$ & $\ldots{ }^{(3)}$ & 1.10 & 0.67 & 1.80 \\
\hline Knot 4 & $37.7-42.4$ & $\ldots{ }^{(2)}$ & $\ldots{ }^{(3)}$ & 0.28 & $0.35:$ & 2.0: \\
\hline
\end{tabular}

-Meaning of columns:

Column (1) - Name of the emission-line feature following the nomenclature of Figure 2.

Column (2) - Region from the long-slit spectrum which was used to calculate the line ratios. The numbers listed in this column represent distances in arcseconds measured west of the nucleus.

Column (3) - Flux ratio $\mathrm{F}([\mathrm{O}$ III $] \lambda 5007) / \mathrm{F}(\mathrm{H} \beta)$.

Column (4) - Flux ratio $\mathrm{F}([\mathrm{O} \mathrm{I}] \lambda 6300) / \mathrm{F}(\mathrm{H} \alpha)$.

Column (5) - Flux ratio F([N II] $\lambda 6583) / \mathrm{F}(\mathrm{H} \alpha)$.

Column (6) - Flux ratio $\mathrm{F}([\mathrm{S} \mathrm{II}] \lambda \lambda 6717,6731) / \mathrm{F}(\mathrm{H} \alpha)$.

Column (7) - Flux ratio F([S II] $\lambda 6717) / \mathrm{F}([\mathrm{S} \mathrm{II}] \lambda 6731)$.

${ }^{(1)}$ Uncertainties on the line ratios range from $10-15 \%$ for the stronger lines to $20 \%$ for ratios involving [S II], and is sometimes as high as $40 \%$ for $[\mathrm{O} \mathrm{I}] / \mathrm{H} \alpha$.

${ }^{(2)} \mathrm{H} \beta$ undetected except perhaps for faint broad emission from knots 3 and 4.

${ }^{(3)}[\mathrm{O} \mathrm{I}] \lambda 6300$ undetected. 


\title{
Artillery Shells over Circinus
}

\author{
Sylvain Veilleux ${ }^{1}$ and Jonathan Bland-Hawthorn ${ }^{2}$
}

Received —_; accepted

\footnotetext{
${ }^{1}$ Department of Astronomy, University of Maryland, College Park, MD 20742; E-mail: veilleux@astro.umd.edu

${ }^{2}$ Anglo-Australian Observatory, P.O. Box 296, Epping, NSW 2121, Australia; E-mail: jbh@aaossz.aao.gov.au
} 


\begin{abstract}
The recently identified Circinus Galaxy is the nearest ( $\sim 4 \mathrm{Mpc})$ Seyfert 2 galaxy known and we now demonstrate to be one of the best laboratories for studying the effects of nuclear activity|3 on the surrounding environment. Here we present new imaging Fabry-Perot observations of Circinus which confirm the existence of an ionization cone in this object but also show for the first time a complex of ionized filaments extending radially from the nucleus out to distances of $1 \mathrm{kpc}$. Arcs suggestive of bow shocks are observed at the terminus of some of these filamentary structures. Most spectacular of all, one of the structures appears to be a scaled-up version of a Herbig-Haro jet. The velocity field of the filaments confirms that they represent material expelled from the nucleus (possibly in the form of 'bullets') or entrained in a wide-angle wind roughly aligned with the polar axis of the galaxy. The motions observed across the ionization cone are highly supersonic, so high-velocity shocks are likely to contribute to the ionization of the line emitting gas. However, it is not clear at present whether shock ionization dominates over photoionization by the Seyfert 2 nucleus. Extrapolation of the filaments to smaller radii comes to within 1" (about 20 pc) of the infrared nucleus, therefore suggesting a AGN or nuclear starburst origin to these features. The complex of radial filaments detected in the Circinus galaxy is unique among active galaxies. The frequency of such events is unknown since only a handful of galaxies have been observed at the sensitivity level of our present observations. The event in the Circinus galaxy may represent a relatively common evolutionary phase in the lives of gas-rich active galaxies during which the dusty cocoon surrounding the nucleus is expelled by the action of jet or wind phenomena.
\end{abstract}

\footnotetext{
${ }^{3}$ In this paper, we use the terms 'nuclear activity' to refer to either starburst or black-hole driven activity in the nuclei of galaxies. Similarly, we refer to 'active galaxies' as galaxies powered by star formation (starburst galaxies) or through accretion onto a massive black hole (active galactic nuclei).
} 
Subject headings: galaxies: active — galaxies: individual (Circinus) — galaxies: jets — galaxies: kinematics and dynamics — galaxies: nuclei — galaxies: Seyfert — galaxies: starburst 


\section{Introduction}

The Circinus galaxy is a large, isolated, gas-rich spiral seen through a relatively unobscured $\left(A_{V} \approx 1.5 \mathrm{mag}\right)$ optical window near the Galactic plane $\left(b=-3.8^{\circ}\right.$; Lyngo \& Hansson 1972 ; Mebold et al. 1976; Freeman et al. 1977). The early detection of strong radio emission from the nucleus (Freeman et al. 1977) provided the first evidence for nuclear activity in this galaxy. Recent maps (Harnett et al. 1990; Elmouttie et al. 1995) have resolved spectacular radio lobes centered on the nucleus, and extending more than $90^{\prime \prime}(\sim 2 \mathrm{kpc})$ on either side of the galaxy disk $\left(\mathrm{PA} \approx-60^{\circ}\right)$. Additional evidence of unusual nuclear activity is suggested by the discovery of an intense 22- $\mathrm{GHz} \mathrm{H}_{2} \mathrm{O}$ megamaser (Gardner \& Whiteoak 1982; Whiteoak \& Gardner 1986). Detailed spectroscopic studies at optical and infrared wavelengths have since revealed the presence of a Seyfert 2 nucleus surrounded by an extended (200-pc radius) circumnuclear starburst (Oliva et al. 1994, 1995; Ghosh 1992; Marconi et al. 1995). A one-sided ionization cone and gas motions indicative of a large central mass concentration have recently been discovered in the Circinus galaxy (Marconi et al. 1995; Greenhill et al. 1997), making it the closest galaxy where the engine responsible for the nuclear activity can convincingly be attributed to a supermassive black hole surrounded by a thick obscuring screen. In this Letter, we describe new TAURUS-2 and long-slit spectroscopic observations which reveal a complex of radial filaments emanating from the nucleus of this galaxy. We propose two possible scenarios to explain these features and briefly discuss the implications of our new results.

\section{Observations}

The Circinus galaxy was observed for 165 minutes on the night of February 211995 using the TAURUS-2 imaging Fabry-Perot interferometer at the AAT 3.9-m telescope. This instrument was used in the Angstrom imaging mode to maximize sensitivity to faint line emission (Bland-Hawthorn et al. 1997). A $40 \mu \mathrm{m}$ gap etalon was used out of band to produce deep, low-resolution ( $~ 350$ $\mathrm{km} \mathrm{s}^{-1}$ sampled at every $\sim 30 \mathrm{~km} \mathrm{~s}^{-1}$ ) [O III] $\lambda 5007$ spectra at each point across this galaxy. 
Each square pixel subtended $0{ }^{\prime \prime} 315$ on the sky; the atmospheric seeing at FWHM averaged approximately 4 times this value. These data comprise kinematic and photometric information for the $\left[\mathrm{O}\right.$ III] line at $\sim 10,000$ positions over the central $2^{\prime}$ of the Circinus galaxy. At a later stage, Circinus was observed at several other etalon spacings to derive the distribution of $\mathrm{H} \alpha$ emission and construct line profiles of [S II] $\lambda \lambda 6716,6731$ and [O III $] \lambda 5007$ at $1 \AA$ resolution. Narrow-band filters were also used at different tilt angles to isolate the lines of He II $\lambda 4686$, [S II] $\lambda \lambda 6717,6731$ and $\mathrm{H} \alpha$, and in order to subtract neighbouring continuum emission. The $\mathrm{H} \alpha$ flux map is discussed below, but the rest of these data are to be presented in a more detailed study (in progress).

In addition, S.L. Lumsden kindly obtained long-slit observations for us at two positions in Circinus. The RGO spectrograph on the AAT was used at the Cassegrain $\mathrm{f} / 8$ focus with the $25 \mathrm{~cm}$ camera and the $270 \mathrm{R}$ grating. At a plate scale $0^{\prime \prime} 77 \mathrm{pix}^{-1}$, this set up gave a resolution of $3.4 \AA$ FWHM at $\mathrm{H} \alpha$ and a wavelength coverage of $4770-8260 \AA$. The $2^{\prime \prime}$ slit was somewhat oversized compared to the 1". 1 FWHM seeing. Both slits were aligned at a position angle of $270^{\circ}$, one passing through the nucleus, the other offset $6 . \prime 0 \mathrm{~N}$ of the nucleus. The exposure time for both positions and an offset sky position was 600 sec.

\section{Results}

Figures 1 and 2 show the [O III] and $\mathrm{H} \alpha$ line flux images obtained by integrating the line profiles in the Fabry-Pero data. Only the blueshifted (between -150 and $0 \mathrm{~km} \mathrm{~s}^{-1}$ ) H $\alpha$ emission is presented in these figures to minimize contamination from the (redshifted) circumnuclear emission south-west of the nucleus. These data confirm the existence of a bright ionization cone in Circinus (Marconi et al. 1995), an effect also apparent in our He II and [S II] narrow-band images. But the [O III] data also reveal a number of fainter filaments beyond this cone which cannot be explained by simple illumination effects of an homogeneous environment by an anisotropic (biconical) source of radiation in the nucleus.

The most striking [O III] feature extends along position angle $\sim-50^{\circ}$ spanning a distance of 
$\sim 25-45^{\prime \prime}(500-900 \mathrm{pc})$ from the nucleus. The lateral extent of this filament is near the limit of our resolution ( $\sim 1^{\prime \prime} .5$ after smoothing). This narrow feature is also visible at $\mathrm{H} \alpha$ but with a lower contrast. The gas at this location is highly ionized with a [O III] $\lambda 5007 / \mathrm{H} \alpha$ flux ratio typically larger than unity. Extrapolation of this filament to smaller radii comes to within $1^{\prime \prime}$ (20 pc) of the infrared nucleus (Marconi et al. 1995), suggesting a nuclear (AGN or compact starburst) origin to this feature. A second [O III] filament is also detected, emerging from the nucleus along PA $\approx$ $-120^{\circ}$ out to a maximum radius of $\sim 35^{\prime \prime}(700 \mathrm{pc})$. These radial filaments resemble the optical counterparts of radio jets in more powerful active galaxies (e.g., Sparks, W. B., Biretta, J. A., \& Macchetto, F. 1994).

The most spectacular feature in the $\mathrm{H} \alpha$ data is the hook-shaped filament which extends to $40^{\prime \prime}(800 \mathrm{pc})$ west of the nucleus (Fig. 2). Such features are commonly observed in Herbig-Haro (HH) objects (e.g., HH 47; Hartigan et al. 1993) although have never been seen on galactic scales. The western 'hook' is far more elongated than the wind-blown bubbles in M82 (Bland \& Tully 1988) and NGC 3079 (Veilleux et al. 1994). Additional morphological evidence for outflow exists in the northern portion of our data (Fig. 1). The [O III ] emission along PA $\approx-20^{\circ}$ forms a broad filamentary 'finger' or jet that points back to the nucleus. A knot is present at the tip of this 'finger', $25^{\prime \prime}$ from the nucleus. Bright $\mathrm{H} \alpha$ emission is also visible near this position, the southern portion of which forms a wide $\left(\sim 8^{\prime \prime}\right)$ arc resembling a bow shock. The arc is pointing in the downstream direction consistent with being produced by a collimated jet.

The kinematics derived from the [O III] Fabry-Perot data (Fig. 3) and long-slit spectra (Figs. $4 \& 5)$ bring credence to this nuclear outflow scenario. Non-gravitational motions are observed throughout the [O III] cone, superposed on a large-scale velocity gradient caused by galactic rotation along the major axis of the galaxy $\left(\mathrm{PA}_{\text {maj }} \approx 30^{\circ}\right.$; Freeman et al. 1977). An unusually large velocity gradient of $4 \mathrm{~km} \mathrm{~s}^{-1} \mathrm{pc}^{-1}$ is seen near the position of the bright knot $\sim 12^{\prime \prime}$ from the nucleus along $\mathrm{PA} \approx-30^{\circ}$ (Fig. 3). The side of the knot facing the nucleus presents velocities that are nearly $250 \mathrm{~km} \mathrm{~s}^{-1}$ lower than gas only $3^{\prime \prime}$ north of that position. The emission profiles near that knot are broad $\left(\sim 250 \mathrm{~km} \mathrm{~s}^{-1}\right)$ and perhaps complex. 
The material in the brighter portions of the NW and SW filaments does not seem to take part in the galactic rotation (Fig. 3). The velocities in the NW [O III] filament appear systematically blueshifted by $\sim 0-100 \mathrm{~km} \mathrm{~s}^{-1}$ with respect to the systemic velocity (439 $\mathrm{km} \mathrm{s}^{-1}$; Freeman et al. 1977), while the velocities of the gas in the SW filament are roughly systemic within the errors of the measurements. Non-gravitational motions are also detected along the western 'hook' feature (Fig. 4). A velocity gradient of $80 \mathrm{~km} \mathrm{~s}^{-1}$ over $8^{\prime \prime}\left(0.5 \mathrm{~km} \mathrm{~s}^{-1} \mathrm{pc}^{-1}\right)$ is visible near the location of knot 2 (Fig. 4a). Perhaps the strongest evidence for shocks in our data is seen in knot 4. There, the strong $\mathrm{H} \alpha$ line is blueshifted by $180 \mathrm{~km} \mathrm{~s}^{-1}$ with respect to the [NII] doublet (Figs. 4b \& 5). Velocity shifts between different emission lines are frequently observed in $\mathrm{HH}$ objects and reflect spatially distinct line-emitting regions (e.g., leading edge versus cooling tail; Morse et al. 1994 and references therein). A more detailed analysis of the nuclear long-slit spectra also suggests the presence of broad $\left(\mathrm{FWZI} \approx 800 \mathrm{~km} \mathrm{~s}^{-1}\right.$ ) blueshifted wings in the emission-line profiles produced by knots 2 and 3, but this result needs to be confirmed with spectra of higher signal-to-noise ratio and velocity resolution.

The current radio data also support the existence of a wide-angle outflow in the Circinus galaxy. The NW feature appears to have a radio counterpart at both $13 \mathrm{~cm}$ and $20 \mathrm{~cm}$ (the NW 'plume' in Figs. 2-4 of Elmouttie et al 1995). This appears as a continuum ridge which runs $\mathrm{SE}-\mathrm{NW}$ through the radio map out to $90^{\prime \prime}$ in radius within the bisymmetric lobes. Radio jets in active galaxies are commonly observed to have associated optical emission, but normally confined to an outer surface at the terminus of the shock (Cecil, Bland, \& Tully 1990). The radio data do not have sufficient resolution to argue whether the optical emission fills the NW 'plume' or is confined to the shock front. The $13 \mathrm{~cm}$ radio map also shows a narrow feature which extends due west from the nucleus. At higher resolution, we anticipate that this feature is associated with another jet - distinct from the NW jet - responsible for the western optical 'hook'. Any possible radio counterparts to the SW filament and the northern 'finger' are being masked by strong emission from the galactic disk.

The motions observed across the ionization cone are highly supersonic, so high-velocity 
$\left(V_{s} \gtrsim 100 \mathrm{~km} \mathrm{~s}^{-1}\right)$ shocks are likely to contribute to the ionization of the line emitting gas. Table 1 summarizes the line ratios measured from our long-slit spectra in knots $1-4$ of the western 'hook' and in the nucleus. Large variations are sometimes observed within a single knot. In knot 2, for instance, $[\mathrm{O} \mathrm{I}] / \mathrm{H} \alpha$ falls with radius as $[\mathrm{O} \mathrm{III}] / \mathrm{H} \beta$ increases. This gradient argues against photoionization (of ionization-bounded clouds) by the nucleus, unless the ionization parameter is rising due to a rapidly falling density profile. The relatively constant density-sensitive [S II] $\lambda 6717 / \lambda 6731$ ratio measured at these locations (especially at the two eastern positions in knot 2 ; cf. Table 1, off-nuclear spectrum) rules out this possibility. The very different excitation properties of knots 3 and 4 (cf. Table 1, nuclear spectrum; Fig. 5) are also difficult to explain in the pure nuclear photoionization scenario, although here large variations of the ionization parameter associated with density variations cannot formally be excluded because the $[\mathrm{S} \mathrm{II}] \lambda 6716 / \lambda 6731$ ratio is in the low-density limit and therefore cannot be used as a density indicator. The enhanced $[\mathrm{N} \mathrm{II}] / \mathrm{H} \alpha,[\mathrm{S} \mathrm{II}] / \mathrm{H} \alpha$, and $[\mathrm{O} \mathrm{III}] / \mathrm{H} \beta$ ratios in knots 1 and 2 fall near the range produced by the high velocity photoionizing radiative shocks of Dopita \& Sutherland (1995). However, [O I]/H $\alpha$ is considerably weaker than predicted by the models. Moreover, all of these ratios require large gas velocities $\left(\mathrm{V}_{s} \gtrsim 500 \mathrm{~km} \mathrm{~s}^{-1}\right)$ and therefore imply large projection effects to explain the relatively small apparent velocities in our data. Hybrid models involving fast shocks and power-law photoionization by the Seyfert 2 nucleus (e.g., Viegas-Aldrovandi \& Contini 1989 and references therein) alleviate these problems. Photoionization by the active nucleus of a mixture of ionization and matter-bounded clouds whose relative proportions vary with position in the galaxy may also provide another explanation for the abrupt changes of excitation in the filaments (e.g., Binette, Wilson, \& Storchi-Bergmann 1996).

\section{Origin of the Complex of Radial Filaments}

Jets in active galactic nuclei are attributed to gas centrifugally accelerated along magnetic field lines tied at one end to the accretion disks (Lynden-Bell 1996). The existence of several jets in a single galaxy is difficult to explain in these models unless the active galaxy is host to two or more 
black holes each possessing its own accretion disk and radio jet. A more plausible explanation for the optical filaments and the linear structures observed in the radio map is that they arise from individual mass structures ejected in a wide opening angle possibly from an explosive nuclear event. The apparent extent and velocities of the outflowing gas in the Circinus galaxy suggest that the purported explosive event took place a few million years ago. It is not clear what triggered this event since the Circinus galaxy shows no sign of recent galactic interaction (Freeman et al. 1977). The ejecta from this event have been funneled into a fan with opening angle of $\sim 100^{\circ}$ and symmetry axis along $\mathrm{PA} \approx-65^{\circ}$, i.e. along the direction of the NW radio lobe (Elmouttie et al. 1995). The lack of any optical counterpart to the SE radio lobe argues that the outflow on that side is hidden from view by the inclined $\left(\sim 65^{\circ}\right.$; Freeman et al. 1977$)$ galaxy disk. The symmetry axis of the conical outflow is roughly aligned with the minor axis of the galaxy, and therefore suggests that our line of sight lies only $\sim 15^{\circ}$ outside the cone defined by the outflow.

Hydrodynamical instabilities in the dense shell swept up by an expanding plasmon (Pedlar, Dyson, \& Unger 1985; Taylor et al. 1989) or a time-variable wind (Garcia-Segura, MacLow, \& Langer 1996; Stone, Xu, \& Mundy 1995) offer an alternative explanation for the optical morphology of the Circinus galaxy. Dynamical models (MacLow, McCray, \& Norman 1989; Tomisaka \& Ikeuchi 1988; Suchkov et al. 1994) of wind-blown bubbles have had success reproducing the range of phenomena observed in radio-lobe spirals (Wehrle \& Morris 1988; Wrobel 1994; Veilleux et al. 1994). The outflow event in the Circinus galaxy would fundamentally be driven by this same mechanism, but would correspond to a later stage of evolution when the initial wind-blown shell has broken out and left dense clouds that have since been accelerated and stretched radially by the galactic wind (free wind phase). The impact of a high-speed supersonic wind on dense clouds has been discussed in several contexts including shock-induced star formation (Woodward 1976), supernova blast waves (McKee et al. 1987), and broad-absorption line quasars (Schiano, Christiansen, \& Knerr 1995). Numerical simulations indicate that the initial encounter of the clouds with the wind medium drives a strong shock that may have devastating effects on the cloud structure (Woodward 1976; McKee et al. 1987). Once in ram pressure equilibrium with the wind, however, these clouds may be accelerated up to a significant fraction of the wind velocity before 
Rayleigh-Taylor and Kelvin-Helmholtz instabilities tear them apart (Schiano et al. 1995).

A clue to the origin of the outflow in the Circinus galaxy is provided by the energetics of this event. The mass taking part in this outflow can be estimated from the [O III] $\lambda 5007$ flux. We parametrize the mass in terms of the density, which is poorly constrained, and use an electron

temperature of $10^{4} \mathrm{~K}$. The integrated observed [O III] intensity of $\sim 5 \times 10^{-13} \mathrm{erg} \mathrm{s}^{-1} \mathrm{~cm}^{-2}$ implies a mass of a few times $10^{4} \mathrm{X}^{-1} \mathrm{~N}_{e, 2}^{-1} \mathrm{M}_{\odot}$ where $\mathrm{X}$ is the fraction $(<1)$ of oxygen which is doubly ionized and $\mathrm{N}_{e, 2}$ is the electron density in units of $10^{2} \mathrm{~cm}^{-3}$. In this calculation, we used $\mathrm{A}_{v}=1.5 \mathrm{mag}$, a solar mass fraction for oxygen, and a five-level atom approximation to estimate the emission coefficient (McCall 1984). Taking $200 \mathrm{~km} \mathrm{~s}^{-1}$ as representative for the deprojected gas velocities (a uncertain number; cf. last paragraph of $\S 3$ ), the kinetic energy involved in the NW outflow is a few times $10^{52} \mathrm{~N}_{e, 2}^{-1}$ ergs. A value closer to $\sim 10^{53} \mathrm{~N}_{e, 2}^{-1}$ ergs is probably more representative of the total energy involved in the outflow event since the radio data (Harnett et al. 1990; Elmouttie et al. 1995) suggest that a similar mass outflow is also taking place on the SE side of the nucleus. The energetics of the optical outflow in the Circinus galaxy therefore appear to be relatively modest (equivalent to about 100 supernova explosions if $\mathrm{N}_{e} \approx 100 \mathrm{~cm}^{-3}$ ) and appear to lie at the low energy end of the distribution for wide-angle events observed in nearby galaxies (Cecil et al. 1990; Bland \& Tully 1988; Heckman et al. 1990; Veilleux et al. 1994). This outflow can easily be powered by the AGN or by a compact nuclear starburst. The nuclear starburst detected in Circinus (Oliva et al. 1995) appears somewhat older than the present outflow event, however.

\section{Summary and Implications}

Deep imaging Fabry-Perot data reveal a complex of radial line-emitting filaments in the Circinus galaxy, the closest Seyfert 2 galaxy known. The kinematics of the gas producing these features suggest the ejection of material over a wide opening angle or inhomogeneities in a wide-angle outflow. The proximity of the Circinus galaxy makes it a unique laboratory to study with unprecedented resolution the impact of nuclear winds, supersonic ejecta, and jets on the 
interstellar medium of galaxies. The apparent rarity of radial filaments and bow shocks in active galaxies may reflect the ephemeral nature of this phenomenon or the difficulty in accelerating dense gas clouds coherently. However, few galaxies have been observed with the sensitivity of our present observations. The discovery of these features in the Circinus galaxy, a spiral galaxy with an abnormal richness of gas (Freeman et al. 1977), brings up the possibility that we may be witnessing a common evolutionary phase in the lives of gas-rich active galaxies. Observations of other active galaxies at similar sensitivity will help establish the frequency and duration of this phenomenon. 
We acknowledge useful conversations with Roger Blandford, Pat Hartigan, and Jim Stone on the physics of outflows. We would particularly like to thank S. L. Lumsden for obtaining the long-slit spectra presented in this paper, Keith Jones for helpful comments on the radio data, Brent Tully for the loan of the HIFI etalon, and the referee, E. Oliva, for several suggestions which improved this paper. Parts of this work have been supported by NASA through grant number HF-1039.01-92A awarded by the Space Telescope Science Institute which is operated by the AURA, Inc. for NASA under contract No. NAS5-26555 (SV). 


\section{REFERENCES}

Binette, L., Wilson, A. S., \& Storchi-Bergmann, T. 1996, A\&A, 312, 365

Bland-Hawthorn, J., Meara, S.J.P., Taylor, K., \& Cannon, R.D. 1997, AJ, in press

Bland, J., \& Tully, R. B. 1988, Nature, 334, 43

Cecil, G., Bland, J., \& Tully, R. B. 1990, ApJ, 355, 70

Dopita, M.A., \& Sutherland, R.S. 1995, ApJ, 455, 468

Elmouttie, M., et al. 1995, MNRAS, 275, L53

Freeman, K. C., et al. 1977, A\&A, 55, 445

García-Segura, G., MacLow, M.-M., \& Langer, N. 1996, A\&A, 305, 229

Gardner, F. F., \& Whiteoak, J. B. 1982, MNRAS, 201, 13p

Ghosh, S. K. 1992, ApJ, 391, 111

Greenhill, L. J., et al. 1997, ApJ, 474, L103

Harnett, J. I., et al. 1990, MNRAS, 244, 130

Hartigan, P., Morse, J.A., Heathcote, S., \& Cecil, G. 1993, ApJ, 414, L121

Heckman, T. M., Armus, L., \& Miley, G. K. 1990, ApJS, 74, 833

Lynden-Bell, D. 1996, MNRAS, 279, 389

Lyngo, G., \& Hansson, N. 1972, A\&A Suppl., 6, 327

MacLow, M.-M., McCray, R., \& Norman, M. 1989, ApJ, 337, 141

Marconi, A., Moorwood, A. F. M., Origlia, L., \& Oliva, E. 1995, ESO Mess., 78, 20

McCall, M. L. 1984, MNRAS, 208, 253

McKee, C. F., Hollenbach, D. J., Seab, C. G., \& Tielens, A. G. G. M. 1987, ApJ, 318, 674

Mebold, U., Goss, W. M., van Woerden, H., \& Freeman, K. C. 1976, Proc. astr. Soc. Aust., 3, 72

Morse, J. A., Hartigan, P., Heathcote, S., Raymond, J. C., \& Cecil, G. 1994, ApJ, 425, 738 
Oliva, E., Origlia, L., Kotilainen, J. K., \& Moorwood, A. F. M. 1995, A\&A, 301, 55

Oliva, E., Salvati, M., Moorwood, A.F.M, \& Marconi, A. 1994, A\&A, 288, 457

Pedlar, A., Dyson, J.E., \& Unger, S.W. 1985, MNRAS, 214, 463

Schiano, A. V. R., Christiansen, W. A., \& Knerr, J. M. 1995, ApJ, 439, 237

Sparks, W. B., Biretta, J. A., \& Macchetto, F. 1994, ApJS, 90, 909

Stone, J. M, Xu, J., \& Mundy, L G. 1995, Nature, 377, 315

Suchkov, A. A., Balsara, D. S., Heckman, T. M., \& Leitherer, C. 1994, ApJ, 430, 511

Taylor, D., Dyson, J.E., Axon, D.J., \& Pedlar, A. 1989, MNRAS, 240, 487

Tomisaka, K., \& Ikeuchi, S. 1988, ApJ, 330, 695

Veilleux, S., et al. 1994, ApJ, 433, 48

Viegas-Aldrovandi, S. M., \& Contini, M. 1989, ApJ, 339, 689

Wehrle, A. E., \& Morris, M. 1988, AJ, 95, 1689

Whiteoak, J. B., \& Gardner, F. F. 1986, MNRAS, 222, 513

Woodward, P. R. 1976, ApJ, 207, 484

Wrobel, J. M. 1994, BAAS, 26, 1501 
Fig. 1.- Line flux images of the Circinus galaxy: $a$, [O III] $\lambda 5007$ and $b$, blueshifted (between -150 and $\left.0 \mathrm{~km} \mathrm{~s}^{-1}\right) \mathrm{H} \alpha$. North is at the top and west to the right. The position of the infrared nucleus (Marconi et al. 1995) is indicated in each image by a cross. The spatial scale, indicated by a horizontal bar at the bottom of the [O III] image, is the same for each image and corresponds to $\sim 25^{\prime \prime}$ or $500 \mathrm{pc}$ for the adopted distance of the Circinus galaxy of $4 \mathrm{Mpc}$. The minor axis of the galaxy runs along $\mathrm{PA} \approx 60^{\circ}$ (measured from north to east). The faintest features in the [O III] $(\mathrm{H} \alpha)$ image have a surface brightness of $\sim 4(20) \times 10^{-17} \mathrm{erg} \mathrm{s}^{-1} \mathrm{~cm}^{-2} \operatorname{arcsec}^{-2}$. To suppress the wide dynamic range, the intensity greyscale wraps around and becomes logarithmic at high intensity levels. The radial features along the north (labelled N), north-west, west (labelled W), and south-west axes suggest the ejection of material (possibly in the form of 'bullets') over a wide opening angle or inhomogeneities in a wide-angle outflow. The inclined $\left(\sim 65^{\circ}\right.$; Freeman et al. 1977) galaxy disk hides the south-east portion of this outflow.

Fig. 2.- Line flux images of the western hook-shaped filament: $a$, [O III] $\lambda 5007$ and $b$, blueshifted (between -150 and $0 \mathrm{~km} \mathrm{~s}^{-1}$ ) $\mathrm{H} \alpha$. The position of the infrared nucleus (Marconi et al. 1995) is indicated in each image by a cross. The orientation is the same as in Fig. 1, but the horizontal bar at the bottom of the [O III] image now corresponds to $\sim 250 \mathrm{pc}$. The faintest features in the [O III] $(\mathrm{H} \alpha)$ image have a surface brightness of $\sim 1(4) \times 10^{-16} \mathrm{erg} \mathrm{s}^{-1} \mathrm{~cm}^{-2} \operatorname{arcsec}^{-2}$. Once again, the intensity greyscale wraps around and becomes logarithmic at high intensity levels. The hookshaped filament shares a strong resemblance with HH objects produced by young stars, suggesting a bow-shock origin for this feature. The bow-shock terminus (labelled 4 in the figure) is only detected in $\mathrm{H} \alpha$, but a rough correspondence is observed between the [O III] and $\mathrm{H} \alpha$ knots (labelled $1-3$ ) which delineate the northern edge of this structure. 
Fig. 3.- Velocity field derived from the [OIII] data cube. The velocities range from $170 \mathrm{~km}$ $\mathrm{s}^{-1}$ (white) to $650 \mathrm{~km} \mathrm{~s}^{-1}$ (black). The uncertainties range from about $50 \mathrm{~km} \mathrm{~s}^{-1}$ in the bright line-emitting regions to $100 \mathrm{~km} \mathrm{~s}^{-1}$ or more in the fainter areas. The position of the infrared nucleus (Marconi et al. 1995) is indicated by a cross. The orientation is the same as in Fig. 1, but the horizontal bar at the bottom of the image now corresponds to $\sim 250 \mathrm{pc}$. The large-scale velocity gradient along $\mathrm{PA} \approx 30^{\circ}$ is due to galactic rotation. Superposed on this gradient are non-gravitational motions observed at several locations, including the NW, W, and SW filaments visible in Figure 1 , and a compact region $\sim 12^{\prime \prime}$ from the nucleus along $\mathrm{PA} \approx-30^{\circ}$ coinciding with very strong [O III] emission.

Fig. 4.- Sky-subtracted long-slit spectra. (a) 6!'0 north of nucleus, PA $=270^{\circ}$, (b) through nucleus, $\mathrm{PA}=270^{\circ}$. The vertical bar on the right of each panel represents $\sim 250$ pc. The offnuclear spectrum intersects knots 1 and 2 of Fig. 2, while the nuclear spectrum intersects knots 3 and 4 but only the southern portions of knots 1 and 2. Note the velocity gradient in knot 2 of the upper panel and the strong blueshifted $\mathrm{H} \alpha$ emission in knot 4.

Fig. 5.- Binned spectra at four positions along the slit through the nucleus corresponding to knots 1-4. Each spectrum has been offset by 400 counts. Knots 1 and 2 are binned along the slit over $6^{\prime \prime}$; knots 3 and 4 are binned over $4^{\prime \prime}$. Note the greatly enhanced [N II]/H $\alpha$ ratio along the jet, except on the bow shock at knot 4. Furthermore, the $\mathrm{H} \alpha$ line at knot 4 is blueshifted by $180 \mathrm{~km}$ $\mathrm{s}^{-1}$ with respect to the $[\mathrm{N}$ II] lines. A faint blue wing can be seen on many of the emission lines. 


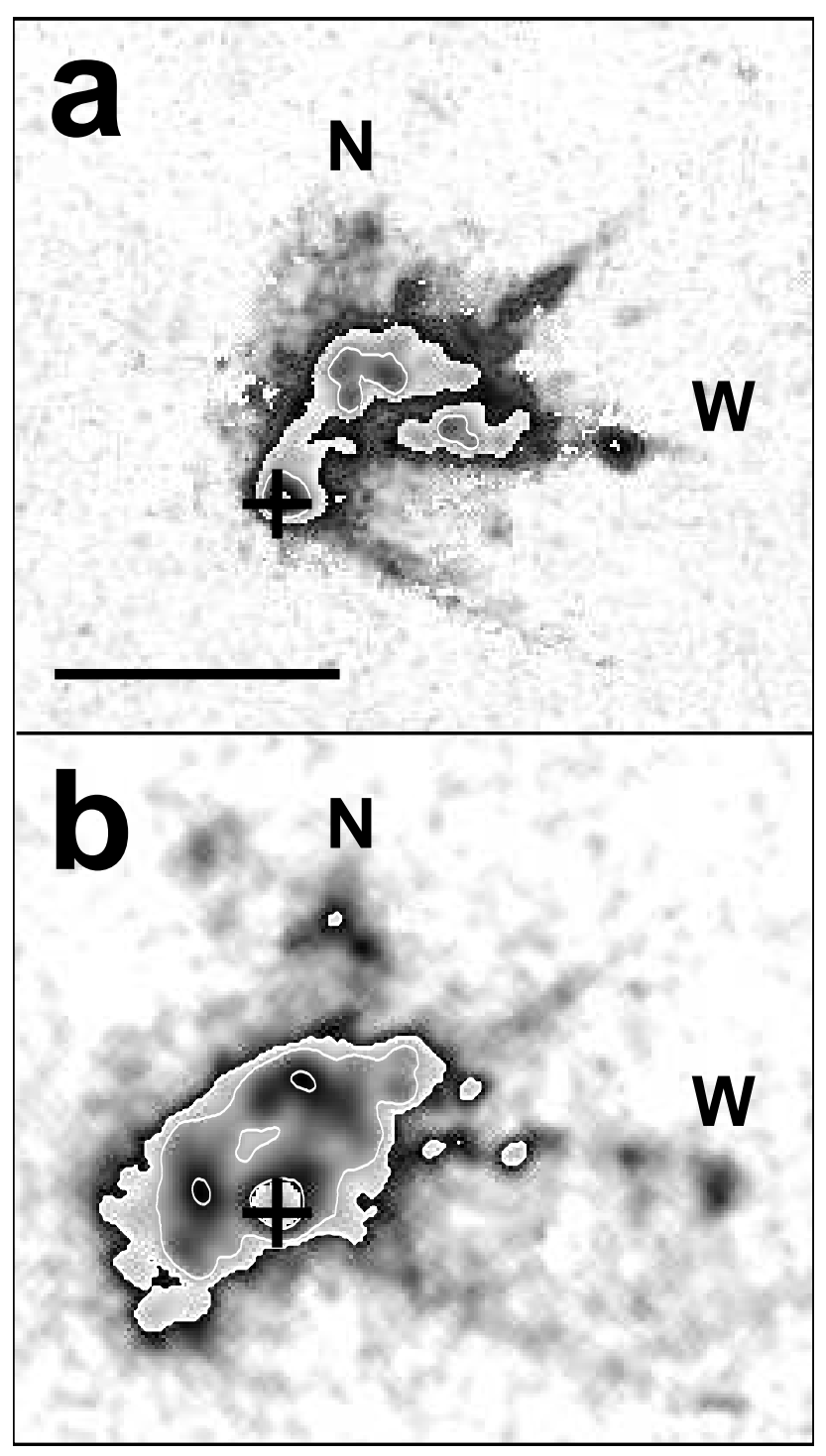

Fig. 1.- 


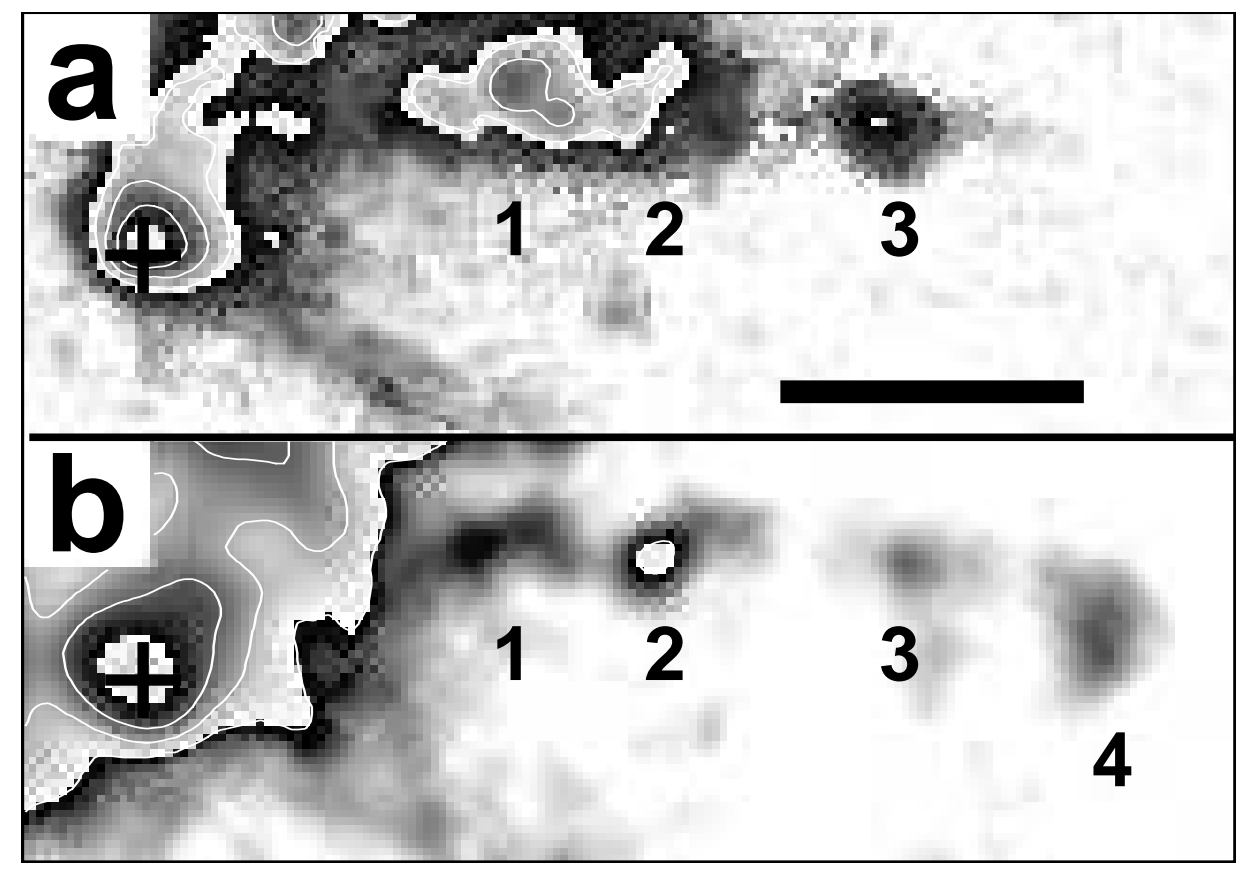

Fig. 2.- 


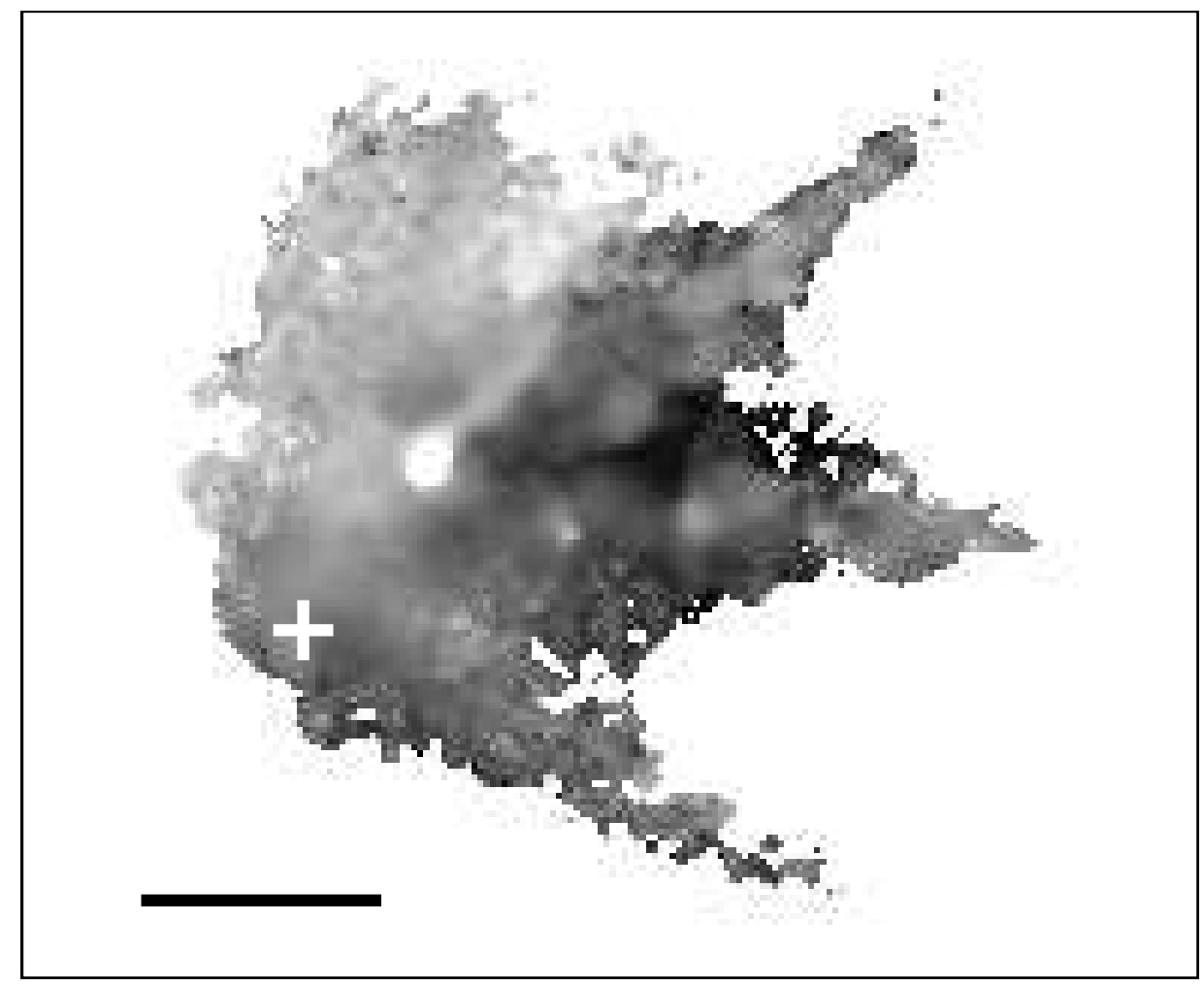

Fig. 3.- 


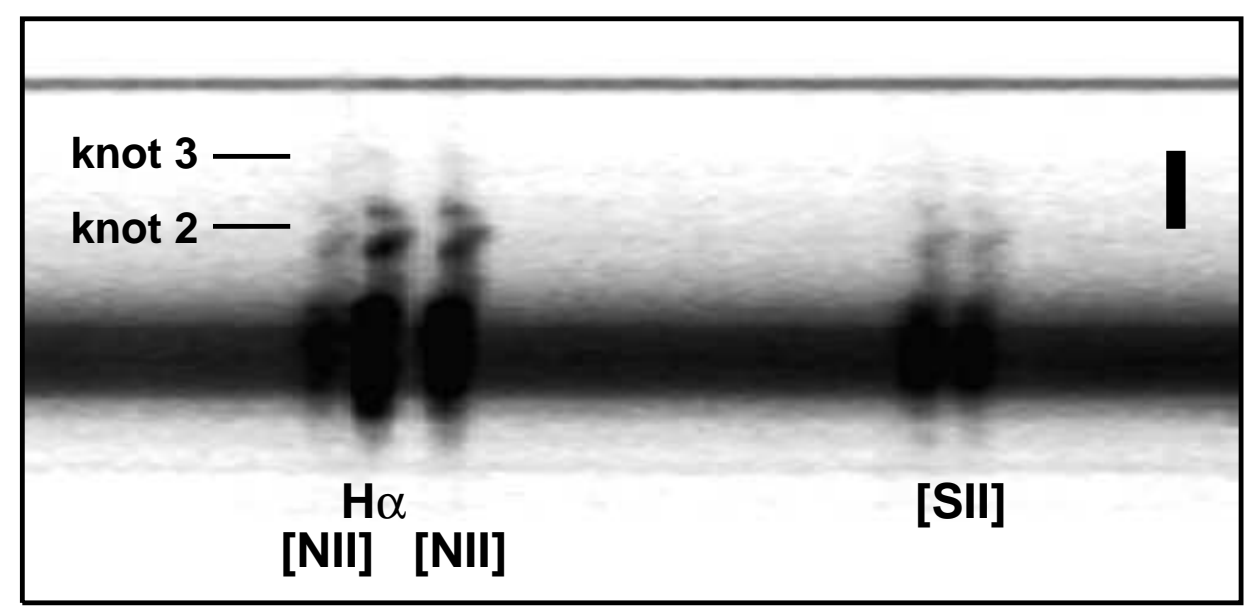

Fig. 4.- a.

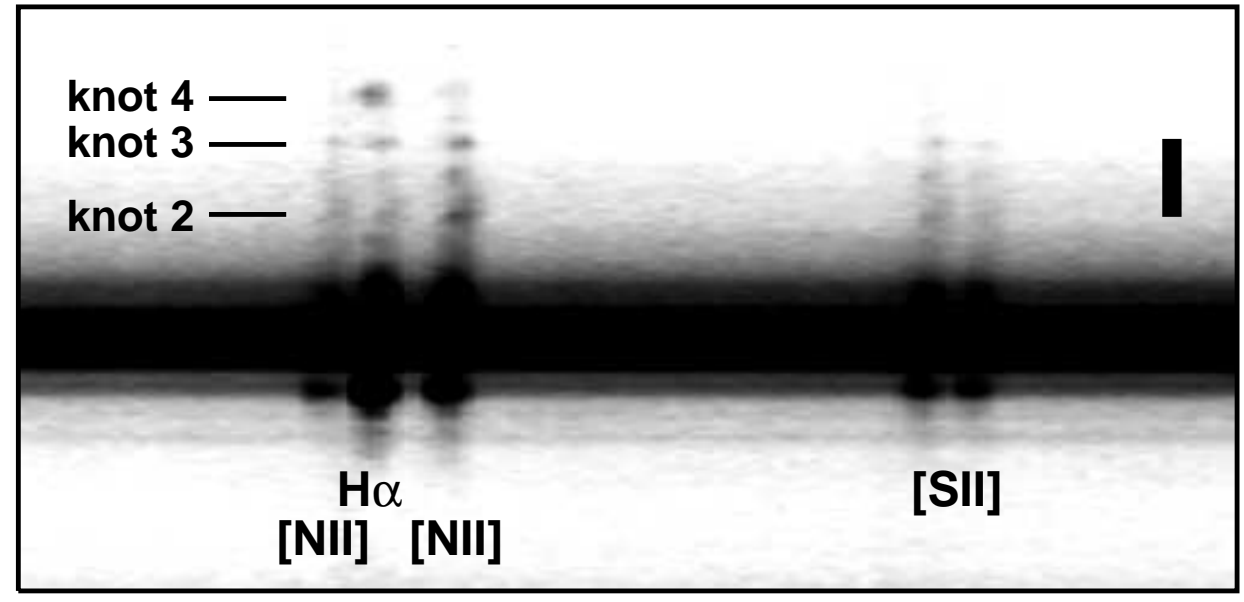

Fig. 4.- b. 


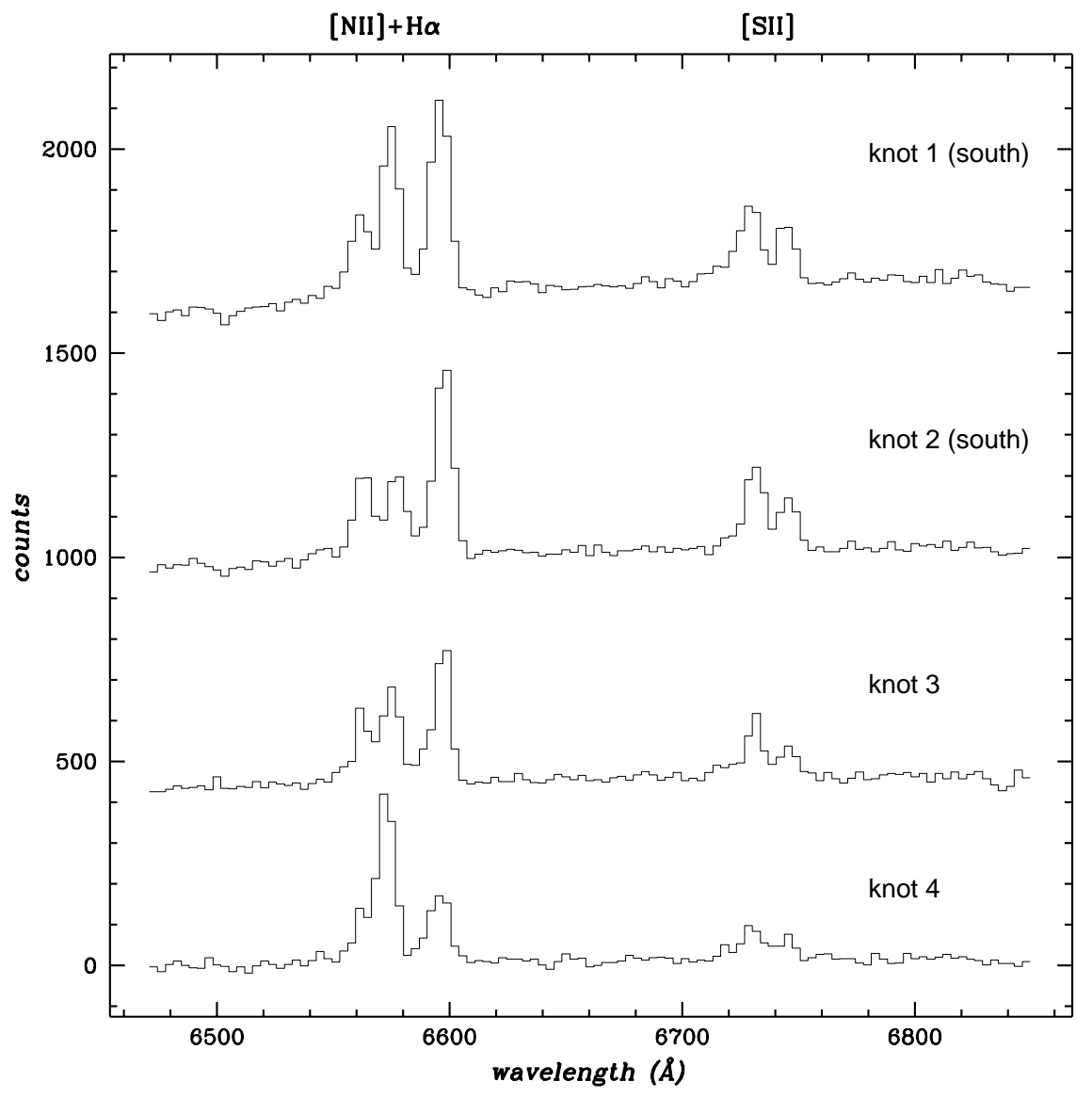

Fig. 5.- 
Table 1: Emission-Line Ratios derived from Long-Slit $\operatorname{Spectra}^{(1)}$.

\begin{tabular}{|c|c|c|c|c|c|c|}
\hline Feature & Region & {$[\mathrm{O} \quad \mathrm{III}] / \mathrm{H} \beta$} & {$[\mathrm{O} \mathrm{I}] / \mathrm{H} \alpha$} & {$[\mathrm{N}$ II $] / \mathrm{H} \alpha$} & {$[\mathrm{S} \mathrm{II}] / \mathrm{H} \alpha$} & [S II] $6717 / 6731$ \\
\hline$(1)$ & $(2)$ & (3) & $(4)$ & $(5)$ & (6) & (7) \\
\hline \multicolumn{7}{|c|}{ Off-Nuclear Spectrum: 6!'0 north of nucleus, PA $=270^{\circ}$} \\
\hline Disk & $-10.0-10.0$ & 8.94 & 0.23 & 0.84 & 0.60 & 1.38 \\
\hline Knot 1 & $10.8-13.9$ & 10.0 & 0.32 & 0.99 & 0.73 & 1.78 \\
\hline Knot 2 & $14.6-16.2$ & 13.9 & 0.23 & 0.89 & 0.58 & 1.22 \\
\hline Knot 2 & $16.9-20.0$ & 16.9 & 0.13 & 0.75 & 0.45 & 1.27 \\
\hline Knot 2 & $20.8-22.3$ & 17.2 & 0.13 & 0.97 & 0.59 & 1.54 \\
\hline \multicolumn{7}{|c|}{ Nuclear Spectrum: through nucleus, $\mathrm{PA}=270^{\circ}$} \\
\hline Nucleus & $-3.9-3.9$ & 13.9 & 0.12 & 1.12 & 0.28 & 1.20 \\
\hline Knot 1 & $11.6-17.7$ & $\ldots^{(2)}$ & 0.13 & 1.41 & 0.93: & 2.0 \\
\hline Knot 2 & $18.5-23.9$ & $\ldots{ }^{(2)}$ & $0.8:$ & 2.00 & 1.42 & 1.85 \\
\hline Knot 3 & $30.8-33.9$ & $\ldots{ }^{(2)}$ & $\ldots{ }^{(3)}$ & 1.10 & 0.67 & 1.80 \\
\hline Knot 4 & $37.7-42.4$ & $\ldots{ }^{(2)}$ & $\ldots{ }^{(3)}$ & 0.28 & $0.35:$ & 2.0: \\
\hline
\end{tabular}

-Meaning of columns:

Column (1) - Name of the emission-line feature following the nomenclature of Figure 2.

Column (2) - Region from the long-slit spectrum which was used to calculate the line ratios. The numbers listed in this column represent distances in arcseconds measured west of the nucleus.

Column (3) - Flux ratio $\mathrm{F}([\mathrm{O}$ III $] \lambda 5007) / \mathrm{F}(\mathrm{H} \beta)$.

Column (4) - Flux ratio $\mathrm{F}([\mathrm{O} \mathrm{I}] \lambda 6300) / \mathrm{F}(\mathrm{H} \alpha)$.

Column (5) - Flux ratio F([N II] $\lambda 6583) / \mathrm{F}(\mathrm{H} \alpha)$.

Column (6) - Flux ratio $\mathrm{F}([\mathrm{S} \mathrm{II}] \lambda \lambda 6717,6731) / \mathrm{F}(\mathrm{H} \alpha)$.

Column (7) - Flux ratio F([S II] $\lambda 6717) / \mathrm{F}([\mathrm{S} \mathrm{II}] \lambda 6731)$.

${ }^{(1)}$ Uncertainties on the line ratios range from $10-15 \%$ for the stronger lines to $20 \%$ for ratios involving [S II], and is sometimes as high as $40 \%$ for $[\mathrm{O} \mathrm{I}] / \mathrm{H} \alpha$.

${ }^{(2)} \mathrm{H} \beta$ undetected except perhaps for faint broad emission from knots 3 and 4.

${ }^{(3)}[\mathrm{O} \mathrm{I}] \lambda 6300$ undetected. 